\title{
Effects of maternal iron status on placental and fetal iron homeostasis
}

\author{
Veena Sangkhae, ${ }^{1}$ Allison L. Fisher, ${ }^{1,2}$ Shirley Wong, ${ }^{1}$ Mary Dawn Koenig, ${ }^{3}$ Lisa Tussing-Humphreys,${ }^{4,5}$ Alison Chu, ${ }^{6}$ Melisa Lelić, \\ Tomas Ganz, ${ }^{1}$ and Elizabeta Nemeth ${ }^{1}$ \\ 'Center for Iron Disorders, Department of Medicine, and ${ }^{2}$ Molecular, Cellular and Integrative Physiology Graduate Program, David Geffen School of Medicine at UCLA, Los Angeles, California, USA. \\ ${ }^{3}$ Department of Women's, Children's and Family Health Science, College of Nursing, ${ }^{4}$ Division of Academic Internal Medicine, Department of Medicine, and ${ }^{5}$ Institute for Health Research and Policy, \\ University of Illinois at Chicago (UIC), Chicago, Illinois, USA. ${ }^{6}$ Department of Pediatrics, Division of Neonatology and Developmental Biology, David Geffen School of Medicine at UCLA, Los Angeles, \\ California, USA. ${ }^{7}$ Medical Faculty, University of Tuzla, Tuzla, Bosnia and Herzegovina.
}

Iron deficiency is common worldwide and is associated with adverse pregnancy outcomes. The increasing prevalence of indiscriminate iron supplementation during pregnancy also raises concerns about the potential adverse effects of iron excess. We examined how maternal iron status affects the delivery of iron to the placenta and fetus. Using mouse models, we documented maternal homeostatic mechanisms that protect the placenta and fetus from maternal iron excess. We determined that under physiological conditions or in iron deficiency, fetal and placental hepcidin did not regulate fetal iron endowment. With maternal iron deficiency, critical transporters mediating placental iron uptake (transferrin receptor 1 [TFR1]) and export (ferroportin [FPN]) were strongly regulated. In mice, not only was TFR1 increased, but FPN was surprisingly decreased to preserve placental iron in the face of fetal iron deficiency. In human placentas from pregnancies with mild iron deficiency, TFR1 was increased, but there was no change in FPN. However, induction of more severe iron deficiency in human trophoblast in vitro resulted in the regulation of both TFR1 and FPN, similar to what was observed in the mouse model. This placental adaptation that prioritizes placental iron is mediated by iron regulatory protein 1 (IRP1) and is important for the maintenance of mitochondrial respiration, thus ultimately protecting the fetus from the potentially dire consequences of generalized placental dysfunction.

\section{Introduction}

Iron is essential for a healthy pregnancy, with approximately 1 gram of iron needed for the expansion of maternal red cell mass and placental and fetal development (1). Severe iron deficiency and iron deficiency anemia during pregnancy have been associated with increased maternal mortality, perinatal death, preterm birth, low birth weight, impaired immune function, and long-term cognitive defects in newborns and infants $(2-6)$. To prevent these adverse outcomes, the WHO currently recommends daily iron supplementation for all pregnant adolescent and adult women (7). On the other side of the spectrum, however, the consequences of high maternal iron levels in pregnancy are not well understood (8). Excess iron can result in the generation of harmful ROS that damage proteins, lipids, and nucleic acids (9), or in increased susceptibility to infection (10). Maternal serum ferritin, a marker of iron stores, has a U-shaped association with adverse pregnancy

Related Commentary: p. 590

Conflict of interest: TC and EN are shareholders of and scientific advisors for Intrinsic LifeSciences and Silarus Therapeutics and are consultants for lonis Pharmaceuticals, Protagonist Therapeutics, Keryx Biopharmaceuticals, La Jolla Pharmaceutical Company, and Vifor Pharma. TC is a consultant for Akebia and Gilead. Copyright: () 2020, American Society for Clinical Investigation. Submitted: January 10, 2019; Accepted: October 22, 2019; Published: December 17, 2019 Reference information: J Clin Invest. 2020;130(2):625-640. https://doi.org/10.1172/JCl127341. outcomes $(11,12)$. However, elevated ferritin is not only a marker of increased iron stores but also of inflammation, and the specific contribution of excess iron has not been resolved. Despite its importance, it is poorly understood how maternal and fetal iron homeostasis during pregnancy is regulated, including the relative contribution of the maternal, placental, and/or fetal signals.

On the systemic level, iron homeostasis is regulated by the hepatic hormone hepcidin, which controls iron absorption and recycling. Hepcidin is induced when systemic iron levels are high and downregulates its receptor, ferroportin (FPN), thereby preventing iron export to blood plasma (13). Thus, changes in hepcidin levels can rapidly modulate plasma iron concentrations. In human pregnancy, maternal hepcidin is suppressed during the second and third trimesters, which is thought to increase iron availability for transfer to the placenta (1). The mechanism of maternal hepcidin suppression is unknown, as is the effect of maternal iron status on this regulatory circuitry.

Iron endowment of the fetus is entirely dependent on iron transport through the placenta. Iron-transferrin (Fe-Tf) from the maternal circulation is taken up by transferrin receptor 1 (TFR1), which is located on the apical surface of the syncytiotrophoblast (14). The complex of Fe-Tf and TFR1 is endocytosed, and iron is released in the endosome and eventually exported on the basal side of the placental syncytiotrophoblast through the iron transporter FPN into the fetal circulation (15). Both TFR1 and FPN are thought to be critical for iron transport across the placenta, as global deletion of either transporter results in embryonic death 
$(16,17)$. Embryonic lethality in TFR1-KO mice occurs before E12.5 (16) and in FPN-KO mice before E9.5 (17). However, the placentaspecific role was only demonstrated for FPN, in which preservation of FPN expression in the placenta but not the embryo proper rescued embryonic lethality (17). The regulation of iron transporters in normal pregnancy or in pathologic conditions such as iron deficiency, iron restriction, or inflammation has not been extensively explored. It was reported that expression of placental TFR1 increased in response to maternal iron deficiency in humans and animal models $(18,19)$, and this was interpreted as a compensatory mechanism to ensure iron delivery to the fetus. Our data refine this model and suggest that both TFR1 and FPN are subject to regulation during iron deficiency, particularly in its more severe forms.

On the cellular level, iron homeostasis is regulated by ironregulatory proteins 1 and 2 (IRP1 and IRP2), which control the stability or translation of mRNAs containing iron-responsive elements (IREs) on their $3^{\prime}$ or $5^{\prime}$ termini, respectively (20). During cellular iron deficiency, IRPs bind to IREs, repressing the translation of mRNAs encoding proteins involved in cellular iron export (FPN) or storage (ferritin) and stabilizing mRNAs encoding proteins involved in iron uptake (TFR1, DMT1), thus maintaining cellular iron levels in the face of iron deficiency. Double IRP1 and IRP2 deficiency is embryonically lethal, whereas mice with the individual IRP KO are viable (21), but the role of these IRPs in regulating materno-fetal iron transfer has not been explored.

We studied mouse models of pregnancy over its time course, as well as human pregnancies and primary human trophoblast cells (PHTs) in vitro, and examined the mechanisms by which maternal iron status (iron-replete, iron-deficient, and iron-loaded) affects the transfer of iron from the mother to the fetus. We found that maternal hepcidin, through regulation of maternal plasma iron concentrations, determines the amount of iron taken up by the placenta and protects the fetus from iron excess even when the mother is iron overloaded. However, we were surprised to discover that during severe maternal iron deficiency, the placenta downregulates FPN to maintain its own iron homeostasis, resulting in decreased iron availability for transfer to the fetus. We devised the placental iron deficiency index (PIDI), a ratio of placental FPN and TFR1, as a sensitive and biologically relevant measure of iron deficiency of the materno-fetal unit. We present evidence that placental iron homeostasis is mediated by the IRE/IRP system to maintain placental function including functional preservation of the placenta's mitochondrial electron transport chain (ETC).

\section{Results}

Effect of maternal iron status on hepcidin and iron availability during pregnancy. In humans, the iron regulatory hormone hepcidin is suppressed during the second and third trimesters of pregnancy (22); however, the mechanisms driving hepcidin suppression remain unknown. To determine whether maternal hepcidin suppression is a consequence of changes in maternal iron status, we assessed suppression in iron-deficient, iron-replete, and iron-loaded WT C57BL/6 dams. Female mice between 6 and 8 weeks of age were either fed a standard chow diet (185 ppm iron) or a low-iron diet (4 ppm iron) 2 weeks prior to and throughout pregnancy, or were injected with 20 $\mathrm{mg}$ iron dextran at the time of mating (Figure 1A). Pregnant animals were analyzed on E12.5, E15.5, and E18.5 (gestation in C57BL/6 mice is approximately 19 days). Females that were subjected to the same iron regimen but did not get pregnant were used as controls and were euthanized on the same day as the pregnant dams. In nonpregnant females, expression of hepcidin (Hamp) mRNA and protein (Figure $1, \mathrm{~B}$ and $\mathrm{C}$ ) changed as expected, depending on the iron status of the mice: iron deficiency lowered hepcidin and iron loading increased hepcidin levels compared with the iron-replete group (serum hepcidin 19,83 , and $346 \mathrm{ng} / \mathrm{mL} ; P<0.001$ deficient vs. replete and $P<0.001$ replete vs. loaded, by 1-way ANOVA and the Holm-Sidak method for multiple comparisons). Compared with nonpregnant females of the same iron status, pregnant females in each group had decreased hepcidin at all time points examined (E12.5-E18.5), replicating the changes observed in human pregnancy (23). Decreased hepcidin was not caused by hemodilution of pregnancy, as equivalent changes were observed for both liver mRNA and serum protein levels (Figure 1, B and C). Importantly, hepcidin suppression was preserved under all maternal iron states: E18.5 serum hepcidin was reduced compared with nonpregnant control levels to $12 \%$ in iron-deficient, $11 \%$ in iron-replete, and $42 \%$ in iron-overloaded conditions (Figure 1C). However, maternal hepcidin was still relatively higher in iron-loaded compared with iron-replete pregnancies, suggesting that iron loading partially counteracts the suppressive pregnancy signal.

Liver nonheme iron concentrations gradually decreased from E12.5 to E18.5 compared with values in nonpregnant control mice, indicating that pregnancy induces iron mobilization from stores (Figure 1D). We surmised that the lowering of hepcidin preceded liver iron mobilization, as at the earliest time point examined, E12.5, hepcidin mRNA and protein levels in iron-replete animals were already nearly maximally suppressed (92\% of maximal serum hepcidin suppression), whereas the decrease in levels of liver iron compared with nonpregnant levels was not yet maximally suppressed at E12.5 (51\% of maximal liver iron suppression) (Figure 1, B-D, middle panels). More important, as a result of decreased hepcidin and iron mobilization from stores, serum iron concentration was maintained throughout pregnancy in iron-replete dams (Figure 1E, middle panel), despite intense utilization of iron for fetal growth during this period. Maintenance of serum iron levels at E18.5 despite low liver stores may be possible because of the relatively high iron content in standard mouse chow.

Unlike iron-replete mothers, both iron-deficient and iron-overloaded mothers were hypoferremic between E12.5 and E18.5 (Figure 1E). This was expected in the iron-deficient group, as both iron stores and dietary iron content were low and obviously insufficient to maintain serum iron levels, even with profoundly decreased hepcidin. Surprisingly, though, when mothers were iron loaded, serum iron was lower compared with levels in nonpregnant animals $(P<0.001$, by 1 -way ANOVA $)$ and also compared with levels detected in iron-replete pregnant animals $(P=0.028$ for pregnant iron-replete vs. iron-loaded animals, by 2-way ANOVA) (Figure 1E). This is almost certainly due to the relatively higher level of maternal hepcidin at E12.5-E18.5, limiting the release of excess iron from stores (Figure 1, B and C, right panel). Changes in maternal transferrin saturation (Supplemental Figure 1; supplemental material available online with this article; https://doi.org/10.1172/JCI127341DS1) paralleled the changes in serum iron levels. 
A

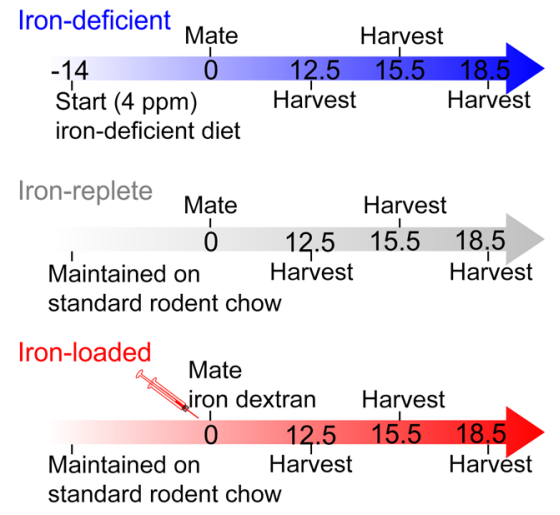

D

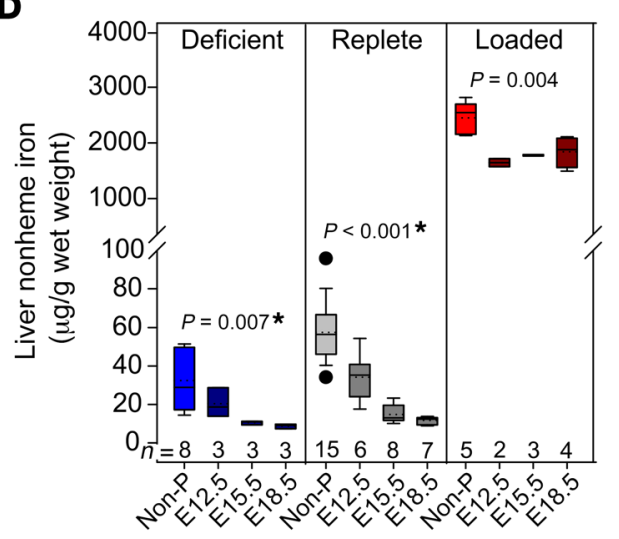

B

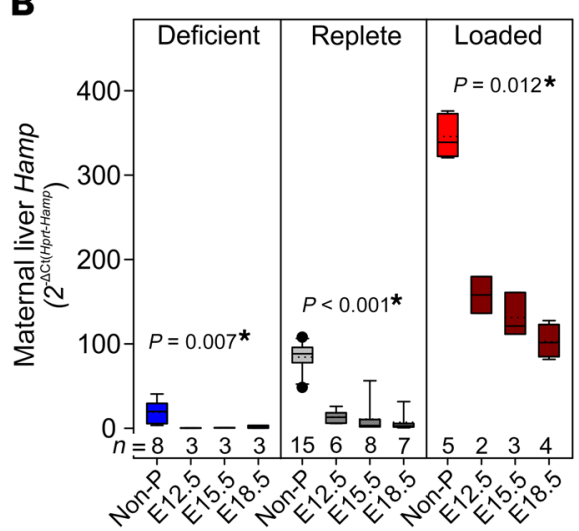

E

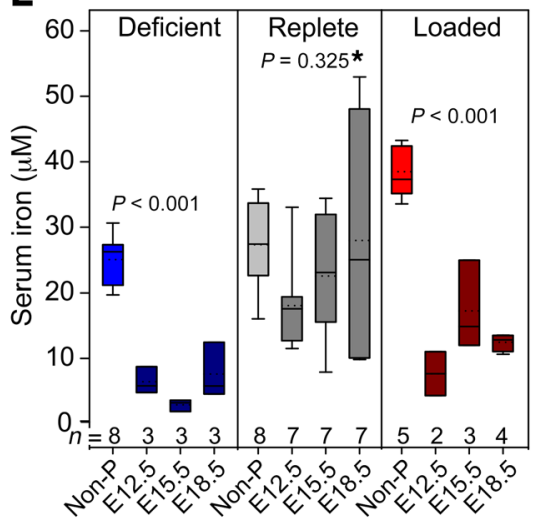

C

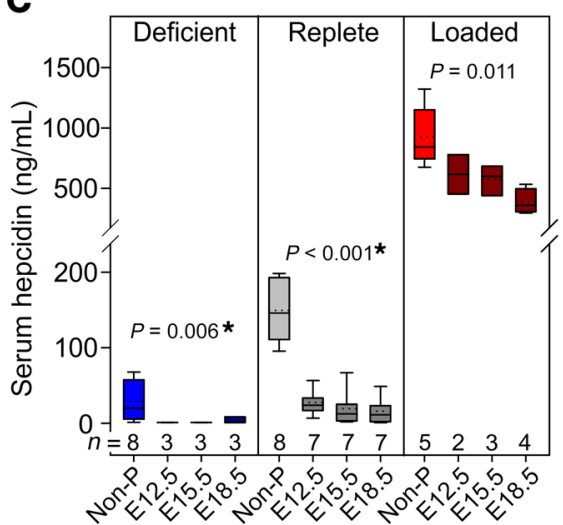

$\mathbf{F}$

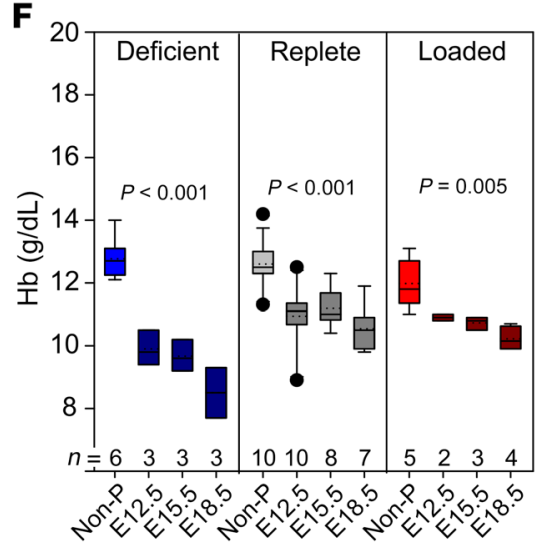

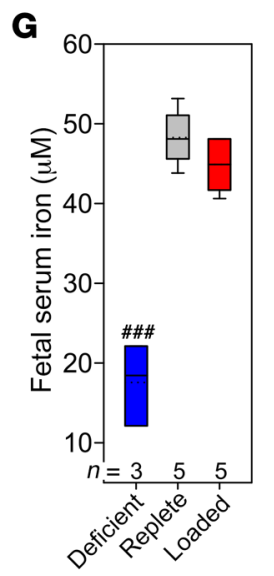
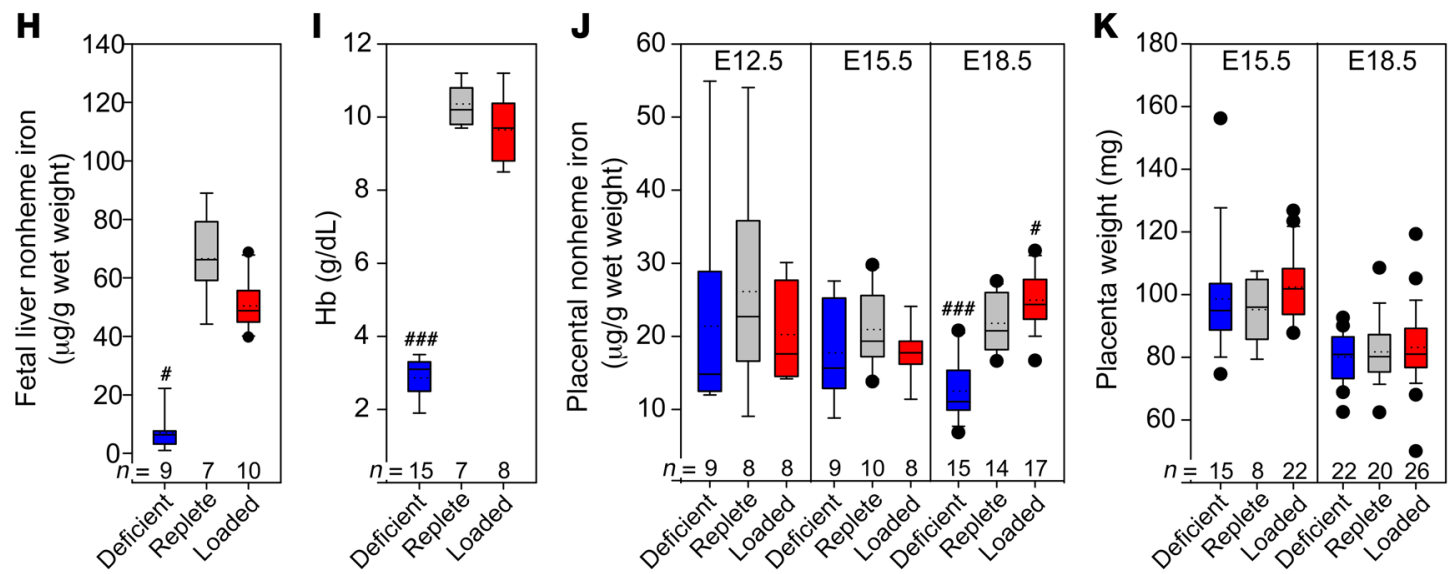

Figure 1. Maternal hepcidin and serum iron levels determine embryo and placental iron status. The iron status of WT C57BL/6 female mice was altered using diet or iron dextran injections. (A) Adult females were fed standard chow ( $185 \mathrm{ppm}$ iron) or a low-iron diet (4 ppm iron) ad libitum 2 weeks prior to and throughout pregnancy, or were injected with $20 \mathrm{mg}$ iron dextran at the time of mating. Pregnant females were analyzed at E12.5, E15.5, and E18.5. Nonpregnant (Non-P) females were subjected to an equivalent iron treatment. (B-F) Maternal measurements of (B) hepcidin (Hamp) mRNA and (C) serum hepcidin. (D) Liver nonheme iron. (E) Serum iron concentration. (F) Hb concentration. Statistical differences between groups was determined by 1 -way ANOVA for normally distributed values, or otherwise by 1-way ANOVA on ranks (indicated by a single asterisk after the $P$ value). (G-I) Embryo measurements at E18.5 for (G) serum iron and (H) liver nonheme iron. (I) Hb concentration. (J) Placental nonheme iron levels at E12.5, E15.5, and E18.5. (K) Placental weight at E15.5 and E18.5 (we did not obtain whole placentas at E12.5). Statistical differences between groups was determined by 1-way ANOVA for normally distributed values followed by the Holm-Sidak method for multiple comparisons versus the iron-replete control group (\#\#\# $P<0.001$ ) or 1-way ANOVA on ranks followed by Dunn's method for multiple comparisons versus the iron-replete control group $(\# P<0.05)$. The numbers of animals are indicated in the $x$ axes of the box and whisker plots.

In iron-replete and iron-loaded pregnant animals, hemoglobin $(\mathrm{Hb})$ concentrations and RBC counts were lower compared with nonpregnant levels, as expected (Figure $1 \mathrm{~F}$ and Supplemental Table 1), because of pregnancy-associated plasma volume expansion. In the iron-deficient group, despite a decrease in hepcidin to nearly undetectable levels, mothers developed frank iron deficiency anemia with decreased $\mathrm{Hb}$ (diet, $P<0.001$ ), RBC counts (diet, $P=0.002$ ), mean corpuscular volume (MCV) (diet, 
$P<0.001)$, and mean corpuscular $\mathrm{Hb}(\mathrm{MCH})$ (diet, $P=0.016$ for iron-deficient vs. iron-replete mice, by 2-way ANOVA) (Figure $1 \mathrm{~F}$ and Supplemental Table 1).

Maternal regulation of iron bioavailability prevents embryonic iron overload but does not protect the embryo from iron deficiency. To determine the consequences of varied maternal iron status for the embryo and placenta, we measured embryonic hematological and iron parameters for pregnancies at E18.5 (see Figure 1). Embryos from iron-deficient mothers were severely iron deficient, as reflected by the decrease in serum and liver iron concentrations (Figure 1, G and $\mathrm{H}$ ). Additionally, brain and whole embryo iron concentrations were decreased (Supplemental Figure 2, A and B). Furthermore, embryonic anemia was much more severe than that in their mothers (Hb in Figure 1I; complete CBC in Supplemental Table 2). Thus, maternal adaptations to iron deficiency were not sufficient to protect the embryos from severe iron deficiency anemia. Embryos from iron-loaded pregnancies, however, had serum iron, liver iron, and hematological parameters similar to those of embryos from control iron-replete pregnancies (Figure $1, \mathrm{G}-\mathrm{I})$. These data suggest that maternal iron regulatory mechanisms including that involving hepcidin protect embryos from iron overload even when mothers are iron loaded.

We observed similar results when iron deficiency and iron overload were caused by alternative approaches: feeding the mice an iron-deficient diet starting at mating (short-term) rather than 2 weeks prior to mating (long-term), or feeding females a high-iron diet (10,000 ppm) starting 2 weeks prior to pregnancy instead of injecting the females with iron dextran at mating. A short-term iron-deficient diet resulted in changes in maternal, fetal, and placental parameters similar to those seen with longer-term iron deficiency (Supplemental Figure 3 and Supplemental Tables 3 and 4 ), with somewhat less impaired maternal hematocrit levels and MCV. Iron loading through a high-iron diet efficiently increased maternal liver iron compared with levels in iron-replete animals at E18.5 ( $P=0.006 ; t$ test on ranks for iron-replete vs. $10,000 \mathrm{ppm})$. Despite maternal iron loading, serum iron concentrations at E18.5 were not higher than those in iron-replete group $(P=0.709$; 2-tailed $t$ test for iron-replete vs. $10,000 \mathrm{ppm}$ ), presumably because of the relative increase in maternal hepcidin. In a comparison of the 2 iron-loading models, however, we found that dietary iron loading resulted in higher maternal serum iron levels. This in turn resulted in increased placental iron content and mildly increased fetal liver iron, although fetal liver iron content remained comparable to that in iron-replete fetuses $(P=0.981$; 2-tailed $t$ test for ironreplete vs. 10,000 ppm) (Supplemental Figure 3, B, F, and H). This suggests that the mode of iron administration during pregnancy could result in subtle differences in iron bioavailability.

When maternal iron availability is low in mice, placental iron homeostasis is prioritized despite fetal iron deficiency. Iron delivery to the fetus is wholly dependent on transfer from the maternal circulation through the placenta, yet factors affecting this transfer are not fully understood. To evaluate the effect of maternal iron status on placental iron content and transport, we analyzed placentas from the pregnancies depicted in Figure 1 at E12.5, E15.5, and E18.5. Placental nonheme iron in the iron-loaded group was only slightly increased at E18.5 compared with the iron-replete group (Figure 1J). The moderation of fetal overload is likely a consequence of the relatively low serum iron concentrations in the maternal circulation, which are maintained by maternal hepcidin. In iron-deficient pregnancies, placental nonheme iron concentrations were comparable to those for other groups at E12.5 and E15.5 and were mildly decreased at E18.5 compared with placental concentrations in iron-replete or iron-loaded pregnancies (Figure 1J), with no change in placental weight (Figure $1 \mathrm{~K}$ ). The decrease in placental iron concentrations at E18.5 was significantly less than the iron decrease detected in fetal livers or fetal $\mathrm{Hb}$ ( 2 -fold in the placenta compared with a 10-fold decrease in fetal livers and a 3.6-fold decrease in fetal $\mathrm{Hb})$. Furthermore, placental heme iron content measured at E18.5 was not different between iron-deficient and iron-replete pregnancies, whereas fetal liver heme iron at E18.5 was significantly lower in the iron-deficient group (Supplemental Figure 2, C and D). Our data suggest that the placenta prioritizes its own iron homeostasis despite fetal iron deficiency.

The principal transporters mediating cellular iron uptake and efflux, TFR1 and FPN, are abundantly expressed in human and mouse syncytiotrophoblast (Figure 2, A and B). We measured TFR1 and FPN protein levels in placentas at E12.5, E15.5, and E18.5 in iron-deficient, iron-replete, and iron-loaded mouse pregnancies (Figure 2, C and D). Protein levels of both transporters increased with gestational age, presumably to meet the increasing iron needs of the developing embryo. We found that TFR1 expression was maximal by E15.5, whereas FPN expression was maximal at E18.5 (Figure 2, C and D). Interestingly, TFR1 expression was not strongly affected by maternal iron status. During maternal iron deficiency, TFR1 protein levels were moderately increased only at E15.5 compared with levels in the iron-replete group (Figure $2 \mathrm{C})$, but these levels were not significantly higher at the other 2 time points (E12.5 and E18.5). TFR1 expression was not different in the iron-loaded group compared with the iron-replete group at any time point examined. Surprisingly, maternal iron deficiency resulted in significantly decreased FPN protein expression at all time points (Figure 2D), with no difference observed in FPN levels between the iron-replete and iron-loaded groups. Decreased FPN during iron deficiency could compromise iron delivery to the fetus while maintaining placental iron content. This is consistent with our observation that maternal iron deficiency had a more profound impact on fetal iron homeostasis than on the placenta: fetuses had dramatically decreased $\mathrm{Hb}$ as well as decreased nonheme and heme iron content in fetal liver, whereas placental nonheme and heme iron levels were much less affected or unaffected (Supplemental Figure 2, C and D). Regulation of placental iron transporters during iron deficiency suggests the existence of placental iron-sensing mechanisms that maintain placental iron homeostasis to the detriment of fetal iron endowment. Interestingly, unlike TFR1 and FPN, we observed that placental ferritin expression did not increase with gestational age (Supplemental Figure 4) and was more responsive to iron loading than was ferroportin (at E18.5). This could be related to ferritin expression in multiple placental cell types (24), unlike FPN, which is primarily expressed in the syncytiotrophoblast.

We devised the PIDI as a ratio of expression of placental FPN to placental TFR1 protein. A decrease in FPN or an increase in TFR1 or changes in both transporters would lead to a lower PIDI, which would be indicative of placental sensing of iron 

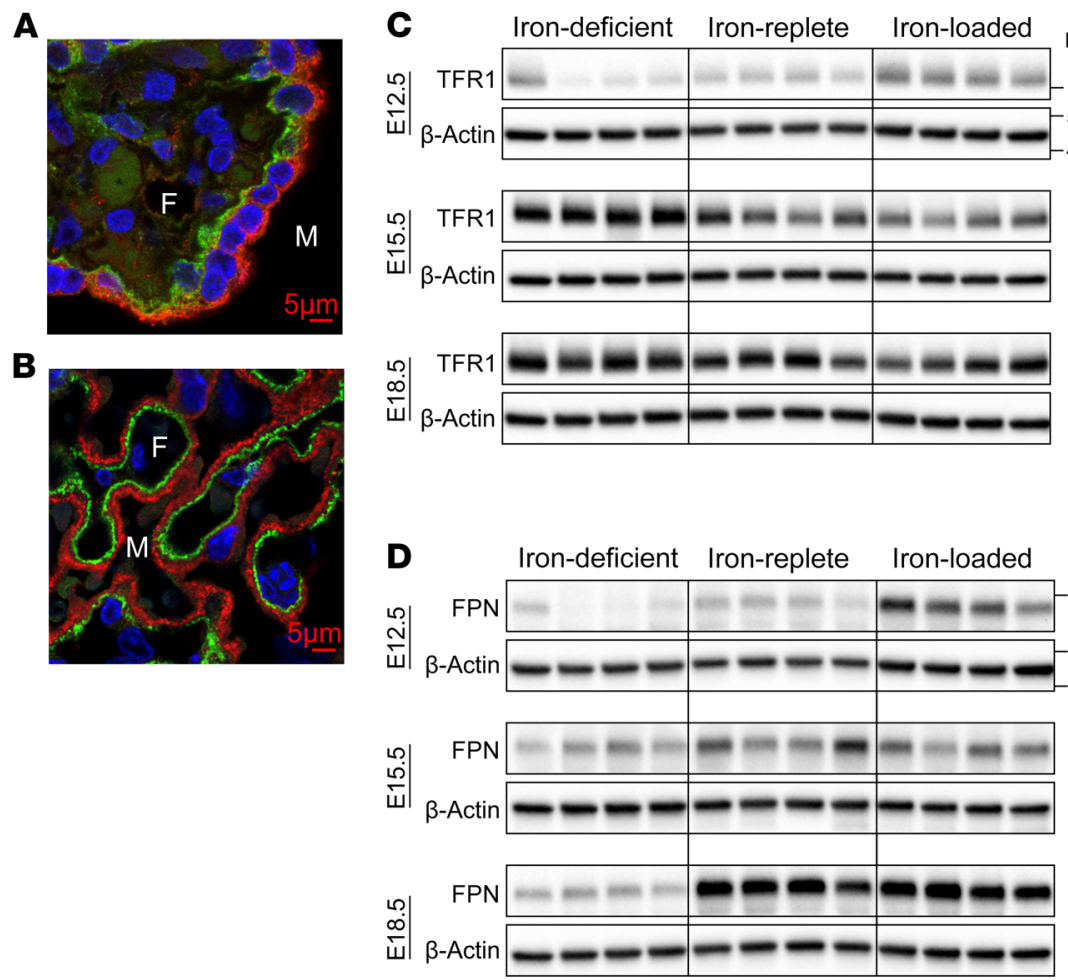
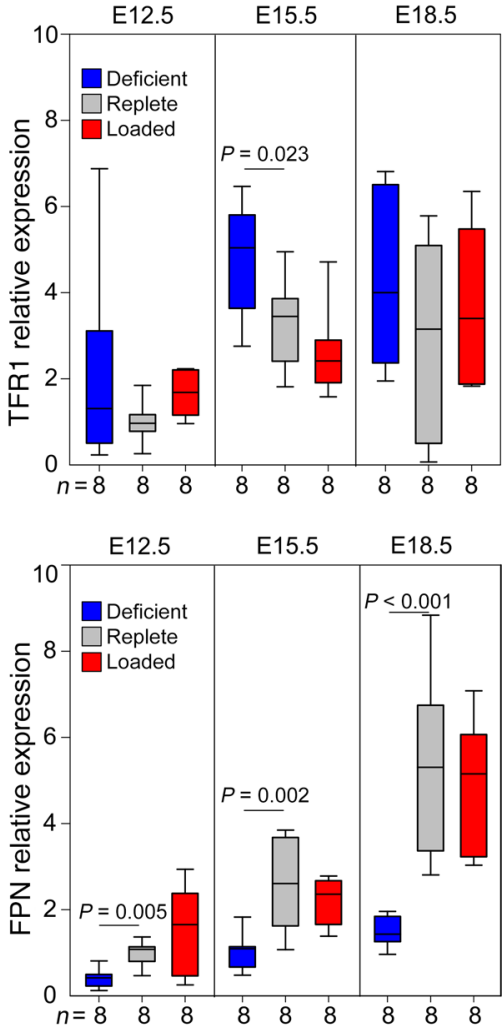

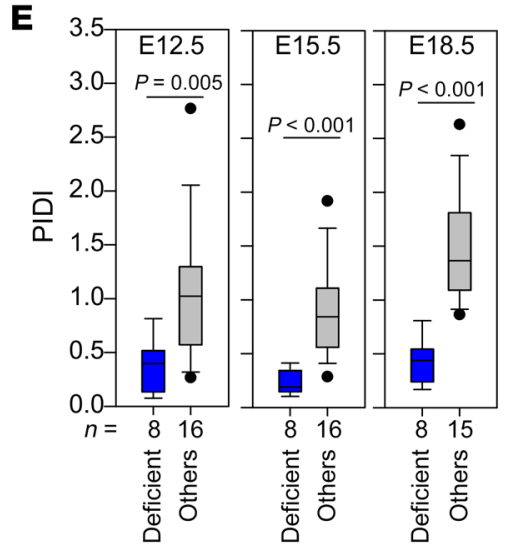

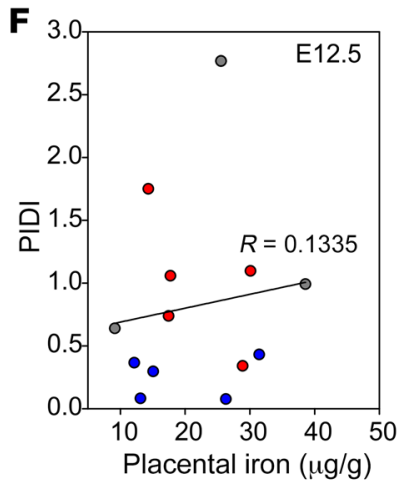

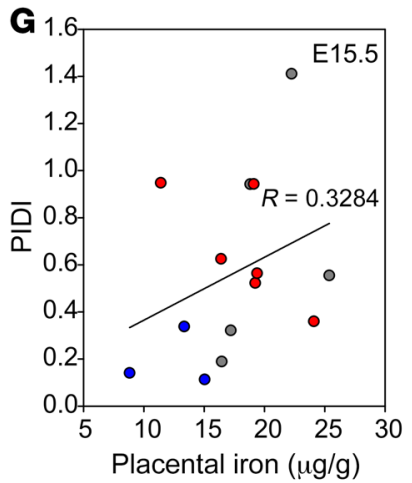

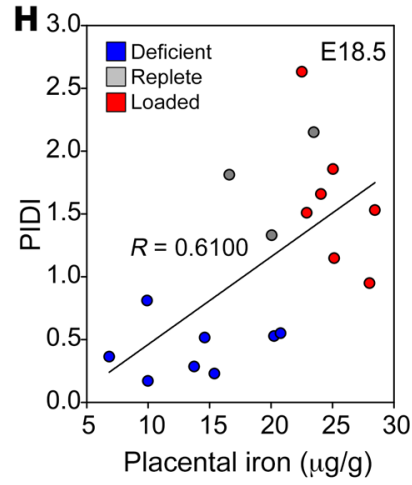

Figure 2. Placental iron transporters respond to changes in maternal iron status. Immunofluorescence staining of human (A) and mouse (B) placentas for TFR1 (red) and FPN (green). Original magnification, $\times 100$. Nuclei are blue. M, maternal circulation; F, fetal circulation. Mouse placentas from Figure 1 were analyzed by Western blotting to determine the protein concentration of TFR1 (C) and FPN (D). $\beta$-Actin was used as a loading control. Four representative placentas are shown in the Western blots, and a total of 8 placentas (from 3 to 4 different dams per group) were used for quantitation. Data are presented as the mean \pm SEM. Statistical differences between groups were determined by 1-way ANOVA for normally distributed values followed by the Holm-Sidak method for multiple comparisons versus the iron-replete control group. (E) The PIDI is the ratio of expression of placental FPN protein to placental TFR1 protein and reflects iron export to the fetus relative to iron import into the placenta from the maternal circulation. Statistical differences were determined using a 2-tailed Student's $t$ test. (F-H) Correlation of nonheme iron with the PIDI at E12.5, E15.5, and E18.5. The numbers of animals are indicated in the $x$ axes of the box and whisker plots.

deficiency and a greater risk of fetal iron deficiency. The PIDI was lower in iron-deficient pregnancies compared with that for iron-replete pregnancies at all 3 time points measured (E12.5, E15.5, and E18.5) (Figure 2E), reflecting a consistent placenta response, whereby iron was prioritized to the placenta, resulting in decreased fetal iron endowment. For assessment of placental iron handling, we argue that using the ratio of FPN to TFR1 in the same placental sample is superior to evaluating single transport- er levels, as the ratio method minimizes the variability in single transporter expression related to placental sampling inconsistencies, which is of particular concern in human placenta analysis.

The correlation between placental nonheme iron levels and the PIDI was marginal at earlier pregnancy time points (E12.5, $R=0.1335$; E15.5, $R=0.3284$ ) (Figure 2, F and G). This is expected, considering that placental iron levels were maintained in the constant range (Figure $1 \mathrm{~J}$ ) by the alterations in 
placental iron transporters (Figure 2, C and D). We observed a stronger positive correlation between placental nonheme iron and the PIDI at E18.5 $(R=0.610)$ (Figure $2 \mathrm{H})$, in which greater placental iron deficiency resulted in a lower PIDI, suggesting decreased iron transfer to the embryo.

To formally test the effect of placental TFR1 and FPN changes on placental iron transfer during maternal iron deficiency in mice, we measured iron transport using a stable Fe isotope. At E17.5, iron-replete and iron-deficient WT C57BL/6J dams received a single i.v. injection of $5 \mu \mathrm{g}$ iron as ${ }^{58} \mathrm{Fe}-\mathrm{Tf}$. Placentas and fetal livers were harvested 6 hours after treatment, and total iron content (nonheme plus heme) in each tissue was measured by inductively coupled plasma-mass spectrometry (ICP-MS) (Figure 3A). We found that injection of ${ }^{58} \mathrm{Fe}$-Tf did not affect placental TFR1 or FPN expression: in iron-deficient pregnancies, placental FPN remained significantly lower than in iron-replete pregnancies (Figure 3B). We measured both ${ }^{56} \mathrm{Fe}$ and ${ }^{58} \mathrm{Fe}$ in the placenta and fetal liver. The ${ }^{56} \mathrm{Fe}$ isotope has the highest natural abundance and provides information on the long-term iron distribution between the placenta and fetal liver.

The short-term ${ }^{58} \mathrm{Fe}$ transport snapshot demonstrated that ${ }^{58} \mathrm{Fe}$-Tf was taken up by the placenta and ${ }^{58} \mathrm{Fe}$ was transferred to the fetus, as it was detectable in the fetal liver. ${ }^{58} \mathrm{Fe}$ did not reach the threshold of detectability when we analyzed whole embryos. In placentas, we observed no statistically significant difference in the amount of total ${ }^{58} \mathrm{Fe}$ retained in iron-deficient versus iron-replete pregnancies, although there was a trend toward approximately $10 \mathrm{ng}$ less ${ }^{58} \mathrm{Fe}$ in the iron-deficient placentas (Figure 3C). In fetal livers, we found that total ${ }^{58} \mathrm{Fe}$ content was significantly lower in iron-deficient pregnancies, with an average of approximately 30 ng lower ${ }^{58} \mathrm{Fe}$ than in iron-replete pregnancies (Figure 3D). These data demonstrate that during iron deficiency, decreased placental FPN results in less iron transport to the fetus. Interestingly, our measurements of total ${ }^{56} \mathrm{Fe}$, which reflects chronic iron transport and handling, showed much greater differences between placental and fetal iron content than did our measurements of ${ }^{58} \mathrm{Fe}$. We found no statistically significant difference in total ${ }^{56} \mathrm{Fe}$ (heme plus nonheme) between placentas from iron-deficient and iron-replete pregnancies $(16.2 \pm 13.2 \mu \mathrm{g}$ and $19.9 \pm 16.1 \mu \mathrm{g}$, respectively) (Figure $3 \mathrm{E})$. However, fetal liver total ${ }^{56} \mathrm{Fe}$ was $68 \%$ lower when mothers were iron deficient $(4.9 \mu \mathrm{g}$ for iron-replete compared with $1.6 \mu \mathrm{g}$ for iron-deficient dams) (Figure 3F). This substantial decrease in fetal liver total iron content despite the stable placental iron content is consistent with results obtained for nonheme iron in our previous experiments. The more profound difference observed in ${ }^{56} \mathrm{Fe}$ content compared with ${ }^{58} \mathrm{Fe}$ could be related to the timing of ${ }^{58} \mathrm{Fe}$ injection, which was near the end of the pregnancy, when the placenta was already fully formed and had acquired most of the iron it needed for its function and was therefore more likely to have sufficient iron for transfer to the fetus.

Regulation of placental iron transporters in human pregnancy. To examine alterations in placental TFR1 and FPN in human pregnancy with varying maternal iron status, we obtained human placental samples at delivery from 39 American women with uncomplicated pregnancies and iron status ranging from normal to moderate iron deficiency. Unlike in our mouse models, iron deficiency in these mothers was not severe enough to cause anemia. TFR1 and
FPN levels were quantified by Western blotting and the PIDI calculated for each sample by dividing normalized FPN with TFR1. Data were grouped by maternal serum ferritin levels, with $10 \mathrm{ng} /$ $\mathrm{mL}$ ferritin used as a cutoff for iron deficiency. We assessed serum ferritin levels in the same women at 32-34 weeks' gestation and at delivery. Regardless of the timing of ferritin assessment (32-34 weeks or at delivery), at the protein level, we found that placental TFR1 was mildly but significantly increased in the $<10 \mathrm{ng} / \mathrm{mL}$ compared with the $>10 \mathrm{ng} / \mathrm{mL}$ ferritin group, consistent with placental sensing of iron deficiency, although the transferrin receptor $(T F R C)$ mRNA differences were not statistically significant (Figure 4, A-D). We also assessed the levels of regnase 1 (REG1), a recently described TFRC mRNA-targeting ribonuclease (25), and found no difference in REG1 mRNA expression between the $<10$ and $>10 \mathrm{ng} / \mathrm{mL}$ ferritin groups (Supplemental Figure 5, A and B). FPN protein levels remained unchanged between the $<10 \mathrm{ng} / \mathrm{mL}$ and the $>10 \mathrm{ng} / \mathrm{mL}$ ferritin groups (Figure $4, \mathrm{E}$ and $\mathrm{F}$ ). The PIDI, the ratio of placental FPN to TFR1, was significantly lower in the $<10 \mathrm{ng} / \mathrm{mL}$ ferritin group than in the $>10 \mathrm{ng} / \mathrm{mL}$ group (Figure 4, $\mathrm{G}$ and $\mathrm{H})$. Placental nonheme iron concentrations were not significantly different between the 2 groups of women (Figure 4, I and J), reflecting a maintenance of placental iron homeostasis. The PIDI appeared to be a superior indicator of even mild iron restriction during pregnancy compared with other parameters commonly measured to assess pregnancy iron status including maternal $\mathrm{Hb}$, cord blood $\mathrm{Hb}$, and cord blood ferritin, which did not differ between the $<10 \mathrm{ng} / \mathrm{mL}$ and $>10 \mathrm{ng} / \mathrm{mL}$ ferritin groups (Figure $4, \mathrm{~K}-\mathrm{O}$ ).

FPN1 mRNA includes isoforms that contain 5 -IREs or not $(26,27)$. Only the $5^{\prime}$-IRE-containing isoform is regulated by intracellular iron levels, as its translation is repressed during iron deficiency. In the human placenta, the $5^{\prime}$-IRE-containing isoform is the predominant FPN mRNA form (Supplemental Figure $5 \mathrm{C}$ ) and is over 10,000 times more abundant than the isoform lacking the $5^{\prime}$-IRE. Therefore, FPN translation would be expected to be inhibited by placental iron deficiency. The lack of FPN protein change between the $<10 \mathrm{ng} / \mathrm{mL}$ and $>10 \mathrm{ng} / \mathrm{mL}$ ferritin groups is likely attributable to the relatively mild iron deficiency of the placentas in our maternal cohort. To determine whether more severe iron deficiency affects FPN protein levels, we analyzed freshly isolated PHTs grown in DMEM and 10\% FBS with addition of the iron chelator desferoxamine (DFO), apo-transferrin (apo-Tf), or holo-transferrin (holo-Tf) for 24 hours. Both DFO and apo-Tf treatment led to a decrease in FPN protein levels compared with the holo-Tf condition (Figure 4P), as well as an increase in TFR1 and a decrease in ferritin levels. We thus surmise that the initial response of the human placenta to mild iron deficiency is to increase TFR1 and that a decrease in FPN only occurs with more severe placental iron deficiency. The mechanism of this differential sensitivity to intracellular iron levels remains to be determined.

Placental IRP1 regulates placental iron transporters in response to changes in maternal iron status. To understand the mechanisms involved in regulating placental iron transporter expression in response to changes in maternal iron status, we used the mouse pregnancy model to analyze the mRNA expression of Tfrc and Fpn, their known regulators, as well as several other transporters and molecules potentially involved in iron homeostasis. We detected 
A

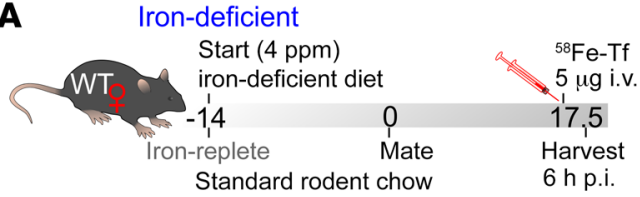

B
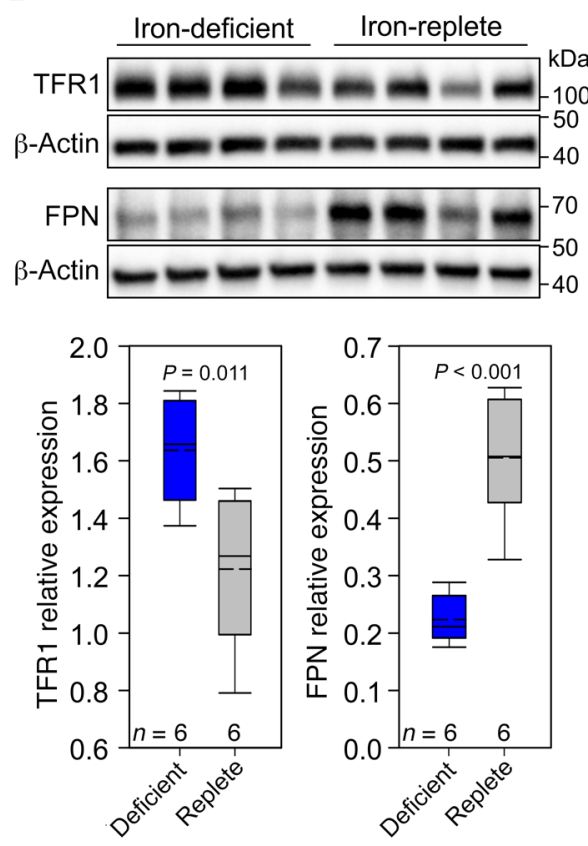

营 ${ }^{\mathrm{T}} \mathrm{TFR} 1$ and FPN protein expression
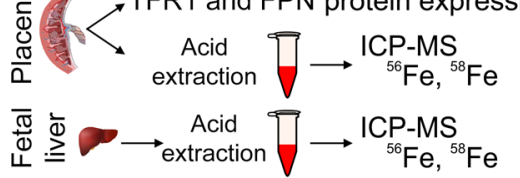

C

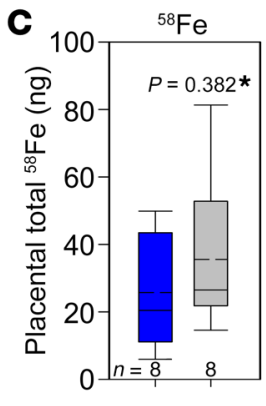

D
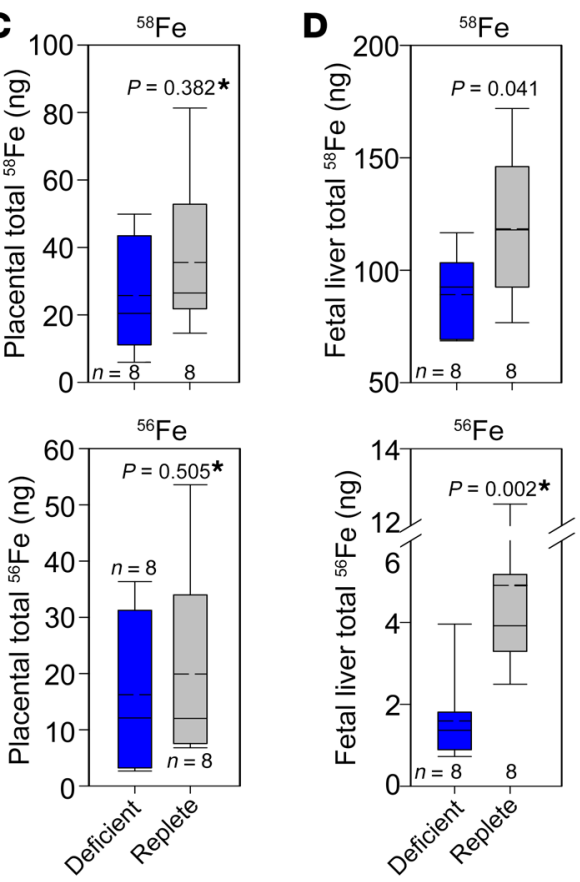

Figure 3. ${ }^{58} \mathrm{Fe}$ transport across the placenta. (A) WT C57BL/6 female mice were maintained on a standard chow diet or placed on an iron-deficient diet 2 weeks prior to mating and were maintained on an iron-deficient diet throughout gestation. At E17.5, the dams received a single i.v. injection of ${ }^{58} \mathrm{Fe}-\mathrm{Tf}$. The dams were sacrificed 6 hours post injection (p.i.), and placental and embryonic tissues were collected and analyzed using ICP-MS. (B) Placental TFR 1 and FPN protein expression was assessed by Western blotting, and quantitation of protein expression relative to $\beta$-actin was performed. Total ${ }^{58} \mathrm{Fe}$ content in (C) placentas and (D) fetal livers. Total ${ }^{56} \mathrm{Fe}$ content in (E) placentas and $(\mathbf{F})$ fetal livers. (B-F) Statistical analysis was performed by 2-tailed Student's $t$ test for normally distributed values and otherwise by Mann-Whitney $U$ rank-sum test (denoted by an asterisk after the $P$ value). The numbers of animals are indicated in the $x$ axes of the box and whisker plots. little change in placental Tfrc mRNA concentrations (Supplemental Figure 6A). In control iron-replete pregnancies, we detected no difference in Tfrc mRNA concentrations between E12.5 and E18.5, suggesting that TFR1 had already reached maximal expression by E12.5. Similar to TFR1 protein results, maternal iron status had small effects on Tfrc levels that were significant only at E18.5 (irondeficient vs. iron-loaded, $P=0.005$, iron-replete vs. iron-loaded: $P=0.005$, by 2-way ANOVA comparison at E18.5). We also found no difference in placental Reg1 mRNA levels related to either gestational age or maternal iron status (Supplemental Figure 6B). To assess Fpn mRNA expression levels, we analyzed 2 known transcript variants of FPN, Fpn1A and Fpn1B (27). As with the human placenta, we found that the iron-regulated, IRE-containing Fpn1A transcript was the predominant isoform in the mouse placenta (Supplemental Figure 6, C and D). Fpn1A increased with gestational age (Supplemental Figure 6C) but was unaffected by maternal iron status (diet $P=0.099$, gestation $P<0.001$, by 2 -way ANOVA). Fpn1B expression was uniformly low, regardless of the gestational age or maternal iron status. We observed no strong iron-dependent difference in placental mRNA expression of the iron transporter divalent metal transporter 1 (Dmt1), homeostatic iron regulator ( $\mathrm{Hfe}$ ), ferroxidase zyklopen (Hephl1), the heme transporter feline leukemia virus subgroup C receptor-related protein 1 (Flvcr1), or the inflammatory markers Il6 and serum amyloid A1 (Saa1) (Supplemental Figure 6, E-J).

The reciprocal change in TFR1 and FPN proteins in response to maternal iron status is consistent with their regulation by the IRE/IRP system, which has been reported to function in human placentas $(28,29)$. Global single IRP1- or IRP2-KO mice are viable, whereas double-IRP1/IRP2-KO embryos die in utero at E6.5 (30). We measured total IRP activity by EMSA and found significantly increased IRP binding in placentas during iron deficiency (Figure $5 \mathrm{~A})$, consistent with decreased FPN protein in the same pregnancies and strongly suggesting that placental iron transporters are regulated by the IRP/IRE system. We were unable to detect any supershift following addition of IRP2 antibody (data not shown), suggesting that IRP1 is the predominant regulator of placental IRE-containing mRNAs. To test the contribution of placental IRP1 to the regulation of placental FPN and TFR1 during maternal iron deficiency, we mated heterozygous IRP1 mice to generate placentas and embryos lacking IRP1 or not. Pregnant dams were placed on a 4-ppm iron diet on E7.5, and placentas were collected on E18.5 (Figure 5B). Loss of IRP1 resulted in loss of placental FPN regulation, and FPN protein was significantly higher during maternal iron deficiency in $\mathrm{Irp1}^{-1-}$ placentas compared with $I r p 1^{+/+}$placentas (Figure 5C). Interestingly, the levels of TFR1 protein or mRNA were not different between $\mathrm{Irpl}^{+/+}$and $\operatorname{Irp1}^{-/-}$placentas (Figure 5, C and D), indicating that Tfrc mRNA is stable even in the absence of IRP1, possibly because of the very low expression of the ribonuclease Reg1 (Figure 5E). We also evaluated whether in IRP1 deficiency, IRP2 may be increased as a compensatory mechanism and stabilize Tfrc mRNA levels. However, we detected no IRP2 activity by EMSA in $\operatorname{Irp1}^{-/-}$placentas (Figure $5 \mathrm{~F})$. Interestingly, there were no detectable differences between $\mathrm{Irp}^{+/+}$and $\mathrm{Irp1}^{-/-} \mathrm{E} 18.5$ embryos in terms of placental or fetal liver iron concentrations (Figure $5, \mathrm{G}$ and $\mathrm{H}$ ), probably because of very low iron availability within the entire materno-placental-fetal unit. At the time of harvesting, the $\mathrm{Irpl}^{+/-}$dams were severely iron 
A TFR1 protein

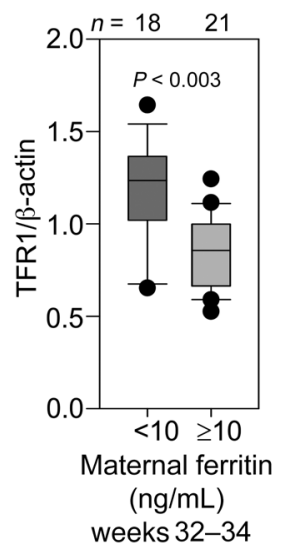

B TFR1 protein

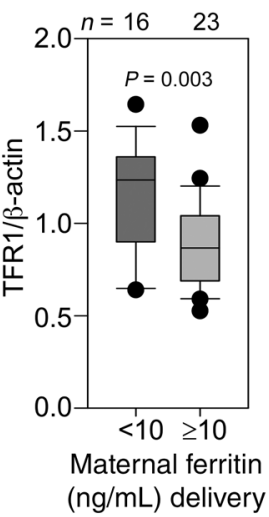

C TFRC MRNA

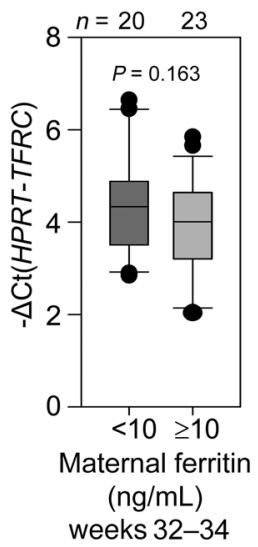

D TFRC MRNA

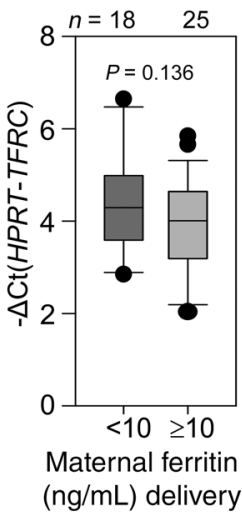

E

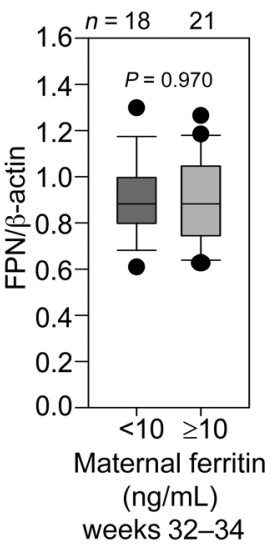

F $\quad$ FPN protein

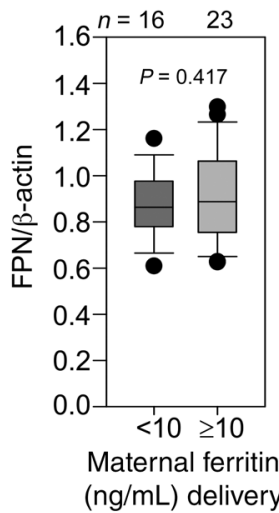

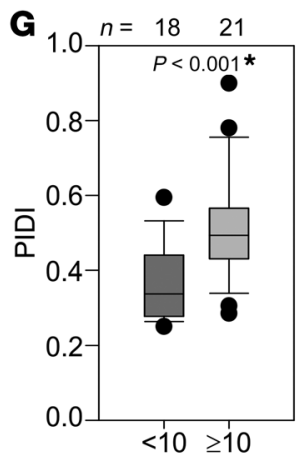

Maternal ferritin (ng/mL) weeks 32-34

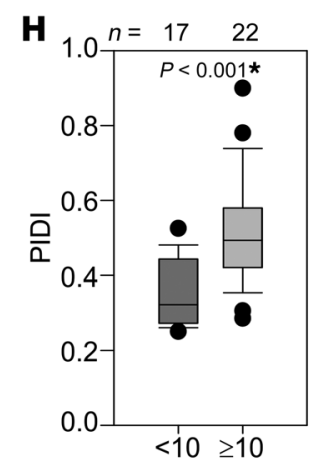

Maternal ferritin ( $\mathrm{ng} / \mathrm{mL}$ ) delivery

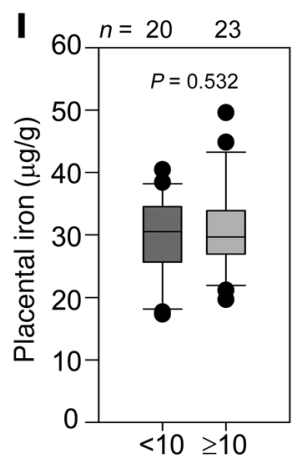

Maternal ferritin (ng/mL) weeks 32-34

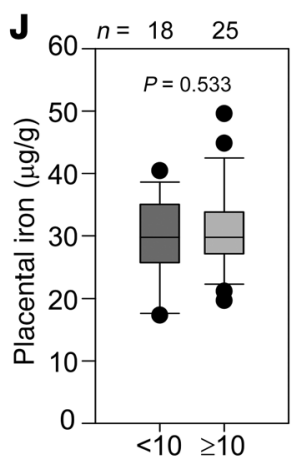

Maternal ferritin ( $\mathrm{ng} / \mathrm{mL}$ ) delivery

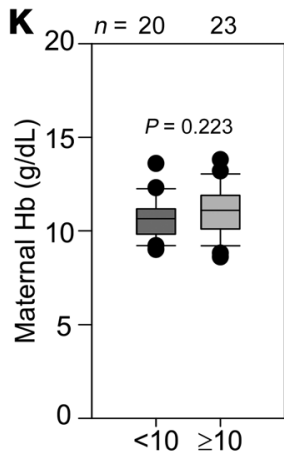

Maternal ferritin (ng/mL) weeks 32-34

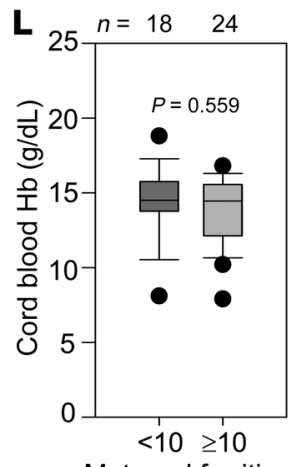

Maternal ferritin (ng/mL) weeks 32-34

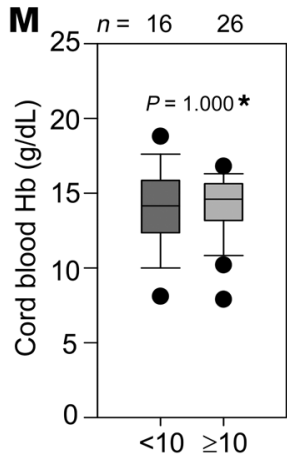

Maternal ferritin $(\mathrm{ng} / \mathrm{mL})$ delivery

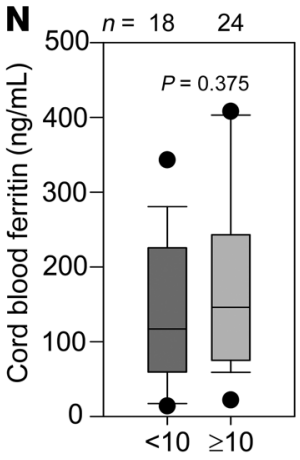

Maternal ferritin (ng/mL) weeks 32-34

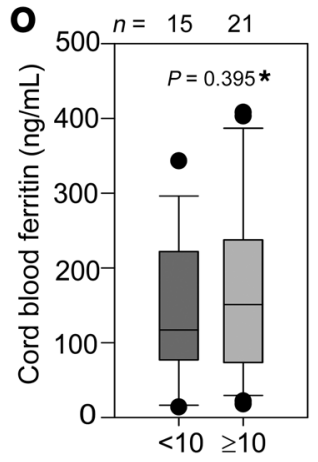

Maternal ferritin ( $\mathrm{ng} / \mathrm{mL}$ ) delivery
$\mathbf{P}$

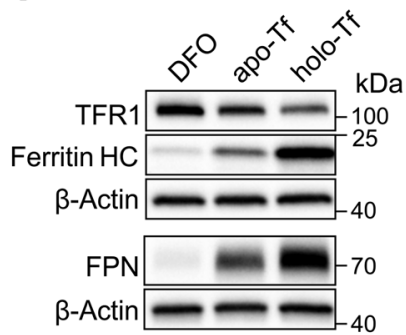

Figure 4. Placental response to maternal iron deficiency in human pregnancy. Placentas from uncomplicated human pregnancies were analyzed by Western blotting to determine protein expression of TFR1 and FPN, normalized to $\beta$-actin. qPCR was performed to determine TFRC mRNA expression, normalized to HPRT. (A and B) TFR1 protein levels, (C and D) TFRC mRNA levels, and (E and F) FPN protein levels according to maternal ferritin during weeks 32-34 or at delivery. (G and $\mathbf{H})$ The PIDI was calculated as the ratio of expression of placental FPN to TFR1 protein, with a lower PIDI reflecting pregnancies at increased risk of fetal iron deficiency. The PIDI was lower in pregnant women with serum ferritin levels below $10 \mathrm{ng} / \mathrm{mL}$ than in those with ferritin levels above $10 \mathrm{ng} / \mathrm{mL}$, regardless of whether ferritin was measured at 32-34 weeks of pregnancy or at delivery. No difference between $<10 \mathrm{ng} / \mathrm{mL}$ and $>10 \mathrm{ng} / \mathrm{mL}$ ferritin groups was observed for (I and J) placental nonheme iron concentrations, (K) maternal $\mathbf{H b}$, (L and $\mathbf{M})$ cord blood $\mathbf{H b}$, or ( $\mathbf{N}$ and $\mathbf{0})$ cord blood ferritin. (P) PHTs were treated with $100 \mu \mathrm{M}$ DFO, apo-Tf or holo-Tf for 24 hours. TFR1, FPN, ferritin heavy chain (HC), and $\beta$-actin expression was assessed by Western blotting. Statistical differences between groups was determined by 2-tailed Student's $t$ test or Mann-Whitney $U$ rank-sum test for non-normally distributed values (denoted by an asterisk after the $P$ value). The numbers of animals are indicated above the box and whisker plots.

deficient (average maternal liver iron, $9.6 \mu \mathrm{g} / \mathrm{g}$ wet weight, $n=5$ ), anemic (average $\mathrm{Hb}, 8.58 \mathrm{~g} / \mathrm{dL}$ ), and hypoferremic (average serum iron, $9.7 \mu \mathrm{M}$ ). Additionally, there could have been a survival advantage for Irp1 $1^{-/}$compared with Irp $1^{+/+}$embryos, as we did not observe the expected Mendelian ratios for embryos from $\mathrm{Irp1}^{+/-} \mathrm{Irp1}^{+/-}$mat- ings when the dams were placed on an iron-deficient diet (WT: $16 \%$, heterozygous: $51 \%$, KO: $33 \%$ ); the differences, however, did not reach statistical significance $\left(P=0.281 ; \chi^{2}\right.$ test, 2 -tailed $P$ value). If IRP1-deficient embryos indeed had a survival advantage, fetal liver iron data for $I r p 1^{1^{++}}$embryos may be skewed, as animals 
A B

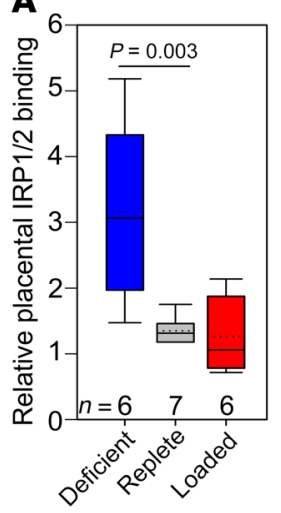

B

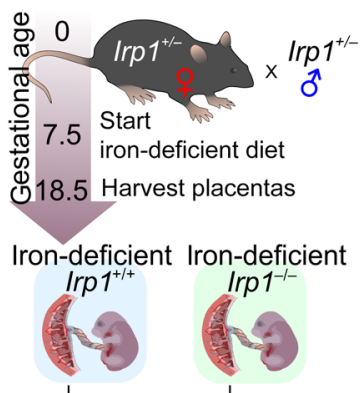

Measure iron transporters

\section{E}
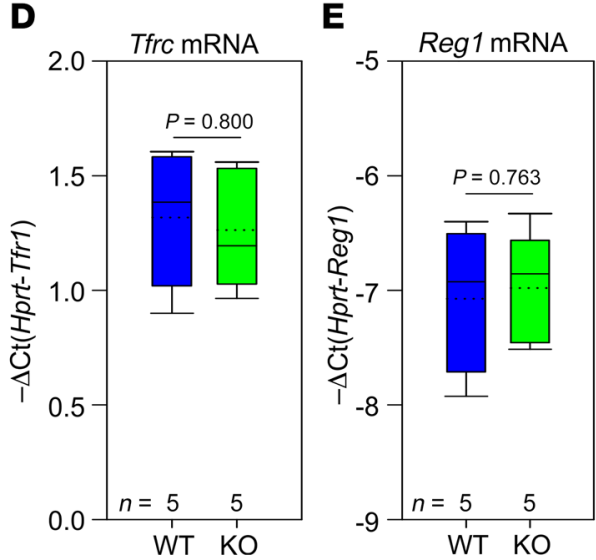

C
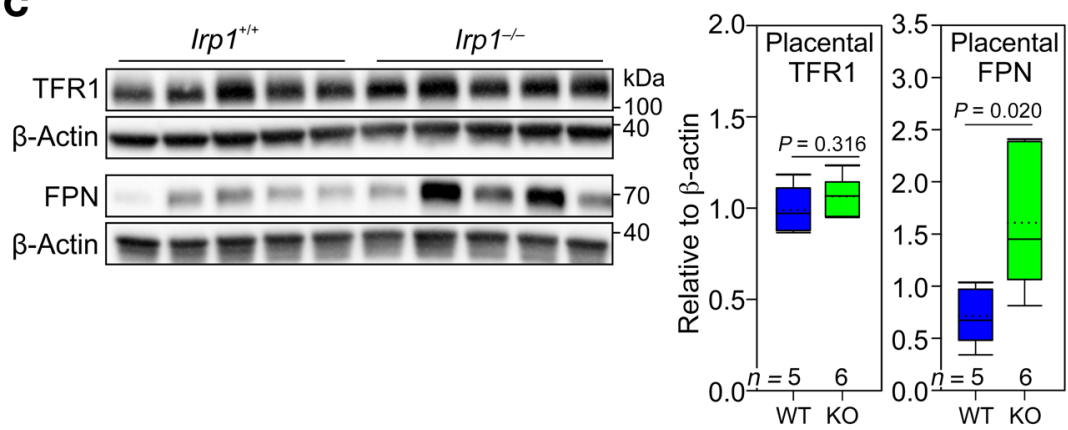

$\mathbf{F}$

Tissue: Liver Irp1 genotype: WT Unlabeled IRE RNA -+ IRP-bound IRE
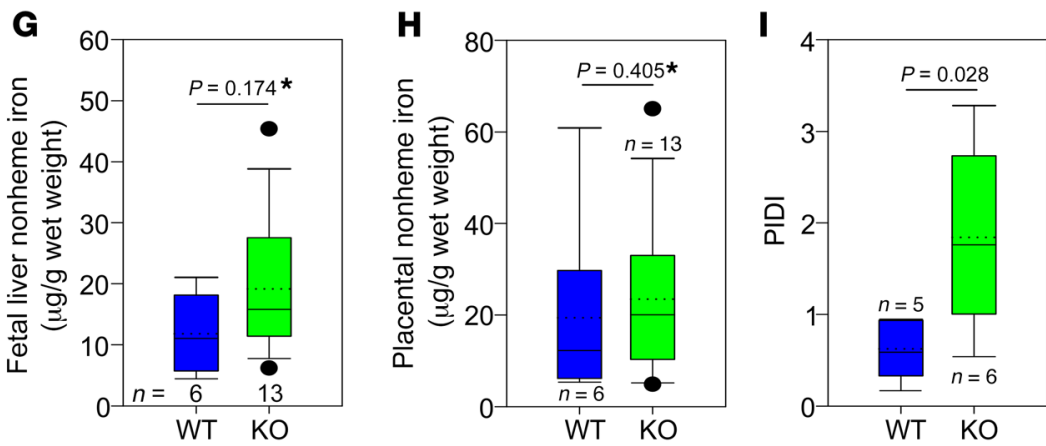

Figure 5. IRP1 mediates placental iron homeostatic responses during maternal iron deficiency. (A) Activity of IRP1/2 in placentas from iron-deficient, iron-replete, and iron-loaded pregnancies was analyzed by EMSA. Statistical differences between groups was determined by 1-way ANOVA on ranks followed by Dunn's method for multiple comparisons versus the iron-replete control group $\left.{ }^{\#} P<0.05\right)$. (B) Irp ${ }^{1+-}$ females were mated with $/ r p 1^{+/-}$males, and pregnant females were fed an iron-deficient diet from E7.5 until E18.5. Placentas and embryos were harvested on E18.5. (C) Placental TFR1 and FPN protein expression was assessed by Western blotting, and quantitation of protein relative to $\beta$-actin was performed. (D) Placental Tfrc mRNA expression.

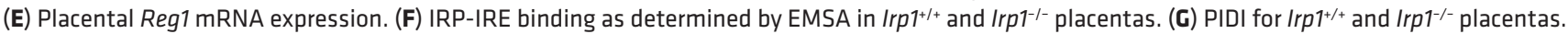

(H) Placental and (I) fetal liver nonheme iron concentrations. (C-I) Statistical analysis was performed by 2-tailed Student's $t$ test for normally distributed values and otherwise by Mann-Whitney $U$ rank-sum test (denoted by an asterisk after the $P$ value). The numbers of animals are indicated in the $x$ axes of the box and whisker plots.

with very low iron levels may not survive. Importantly, however, in the absence of IRP1 regulation (Irp1--), placentas had a higher PIDI (ratio of FPN to TFR1 in each placenta) compared with $\operatorname{Irp1}^{1 /+}$ placentas, confirming that IRP1 is the major regulator of placental iron transporters in response to maternal iron deficiency (Figure 5I).

Placental and fetal hepcidin does not regulate fetal iron endowment. During iron deficiency, local hepcidin regulation was reported to be important for cardiac function (31). In the heart, hepcidin protein was paradoxically increased by iron deficiency, even though its mRNA expression was decreased. If hepcidin protein in the placenta or embryo was also unexpectedly stabilized during iron deficiency, this could account for the decreased FPN protein levels in the placenta. Additionally, placental FPN localizes to the basolateral membrane of the syncytiotrophoblast (14) and thus could be subject to regulation by embryonic hepcidin. In fact, in embryos that overexpress hepcidin either because of hepcidin transgene insertion or Tmprss 6 deficiency, placental FPN is downregulated, resulting in severe fetal iron deficiency $(32,33)$. However, embryonic hepcidin is low in normal mouse pregnancies (32, 33). To test whether the placental response during iron deficiency may be subject to regulation by placental or embryonic hepcidin, we bred heterozygous hepcidin mice and placed the females on either an iron-replete or iron-deficient diet 1 week prior to mating, generating iron-replete or iron-deficient $\mathrm{Hamp}^{+/+}, \mathrm{Hamp}^{+/-}$, or $\mathrm{Hamp}^{-/}$placentas and embryos. As expected, a low-iron diet elicited maternal iron deficiency and anemia (Supplemental Figure 7, A-F), as well as fetal iron deficiency (Figure 6, A and B). However, within the same diet group, we detected no difference in fetal liver iron or serum iron among $\mathrm{Hamp}^{+/}, \mathrm{Hamp}^{+/}$, or $\mathrm{Hamp}^{-/}$fetal genotypes. Placental iron concentrations did not differ between iron-replete and iron-deficient groups, or between different Hamp genotypes (Figure 6C). Placental Hamp mRNA expression was not different between the different diet groups or between WT and heterozygous placentas (Figure 6D). Placental Tfrc mRNA was mildly increased and FPN protein significantly decreased in the iron-deficient group, as expected, but again with no difference observed among the different Hamp genotypes (Figure 6, E and F). The results indicate that loss of placental and embryonic hepcidin has no effect on placental or fetal liver iron status in iron-replete or iron-deficient pregnancies. 
A

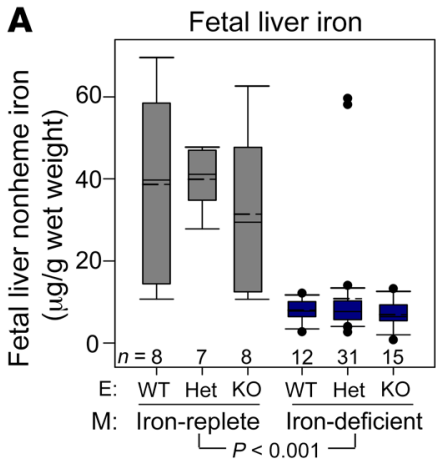

D

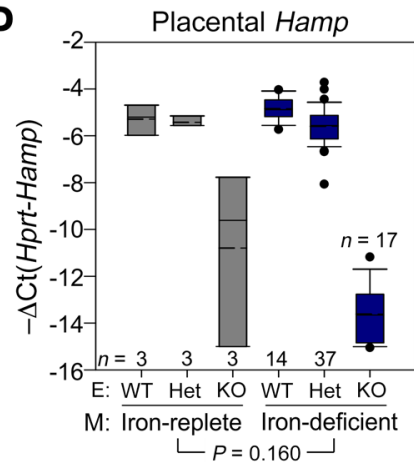

B

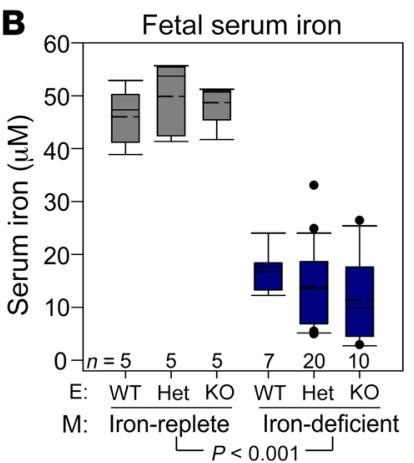

C

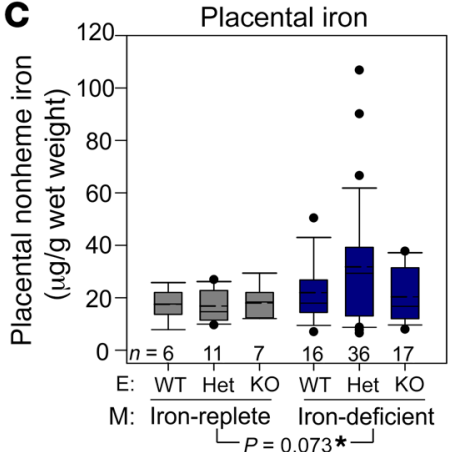

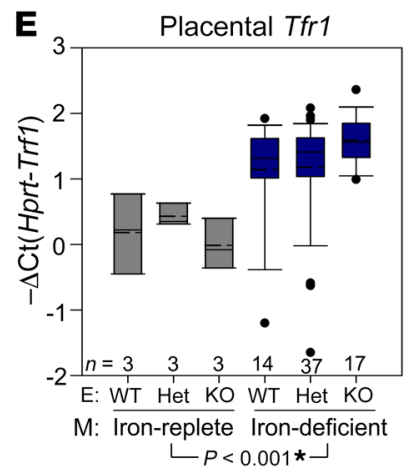
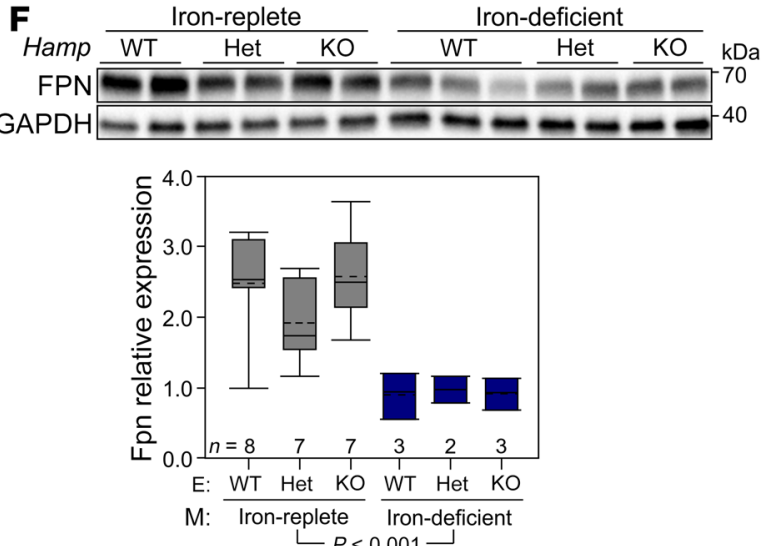

Figure 6. Placental and embryonic hepcidin does not regulate placental iron homeostasis under iron-replete or iron-deficient conditions. Hamp ${ }^{+/-}$ female mice were mated with $\mathrm{Hamp}^{+/-}$male mice. Females were placed on an iron-replete or iron-deficient diet 1 week prior to mating. Placentas and embryos were analyzed on E18.5. (A) Fetal liver iron and (B) fetal serum iron concentrations. (C) Placental iron concentration, (D) Hamp expression, (E) Tfrc expression, and (F) FPN protein levels. Statistical differences between groups were determined by 2-way ANOVA (non-normally distributed values are indicated by an asterisk). The $P$ value shown is for variation by diet. There were no statistical differences between genotypes for any of the measured parameters. The numbers of animals are indicated in the $x$ axes of the box and whisker plots. E, embryos; M, mothers; Het, heterozygous.

We also measured expression levels of the miRNA miR485-3p (34), which was reported to be induced by cellular iron deficiency and mediate suppression of FPN via a posttranscriptional mechanism. However, we found no difference in mouse miR-485-3p expression between iron-deficient and iron-replete pregnancies (Supplemental Figure 8).

Iron is necessary for trophoblast function. The placenta is a highly metabolically active tissue, consuming approximately $40 \%$ of total uterine oxygen uptake (35). This large energy consumption is primarily used for mitochondrial ATP synthesis to support protein production, nutrient transport, and fetal metabolite waste transport (36). To assess the consequences of iron deficiency for the placenta, we measured the concentrations of ETC complexes CI-CV in E18.5 placentas from iron-replete and iron-deficient pregnancies and found no differences in complex expression (Supplemental Figure $9 \mathrm{~A})$. In vivo, however, the placenta is protected from large changes in iron status by its homeostatic response during iron deficiency, i.e., increased iron import through TFR1 and decreased export through FPN. Therefore, we isolated trophoblast cells from term human placentas (PHTs) and cultured them for 24 hours under a range of iron conditions. We used the iron chelator DFO to deplete the cells of iron, holo-Tf to iron load them, and apo-Tf as a baseline control. As shown in Figure 4P, iron deficiency increased the expression of TFR1 and decreased FPN and ferritin. As in mouse placentas, we detected no change in the expression of OXPHOS complexes following iron treatments (Figure 7A), probably because of the protective redistribution of intracellular iron at this time point. We next performed respirometry assays to measure how trophoblast iron levels affect mitochondrial respiration. Iron depletion decreased all the measured bioenergetic parameters of respiration compared with apo-Tf and holo-Tf treatments, with a significantly lower basal, ATP-linked, maximal, and spare respiratory capacity oxygen consumption rate (OCR) in iron-depleted cells (Figure 7, B and C). The reduction in the maximal OCR suggests that iron deficiency may perturb the placenta's ability to generate energy for its synthetic and transport functions. Following inhibition of ATP synthase by oligomycin, PHTs compensated by shifting to glycolysis under all iron conditions (Figure 7D), demonstrating that the cells remained viable. Comparison of the baseline OCR and extracellular acidification rate (ECAR) (Figure 7E) confirmed that iron depletion decreased the OCR but not the ECAR. The uniformly low ECAR indicated that mitochondrial respiration rather than glycolysis is the predominant form of energy production in the trophoblast.

As an alternative trophoblast model, we used BeWo cells, a placental cell line derived from a human choriocarcinoma often used to study placental transport, including that of iron (37). Like PHTs, BeWo cells were treated with DFO, apo-Tf, or holoTf for 24 hours. DFO treatment efficiently depleted cells of iron, 
A

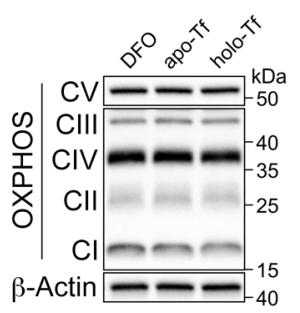

B

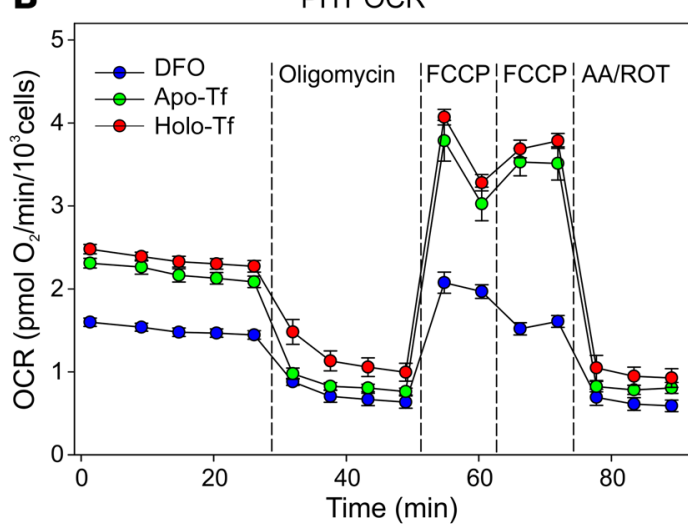

E Basal OCR vs, basal ECAR
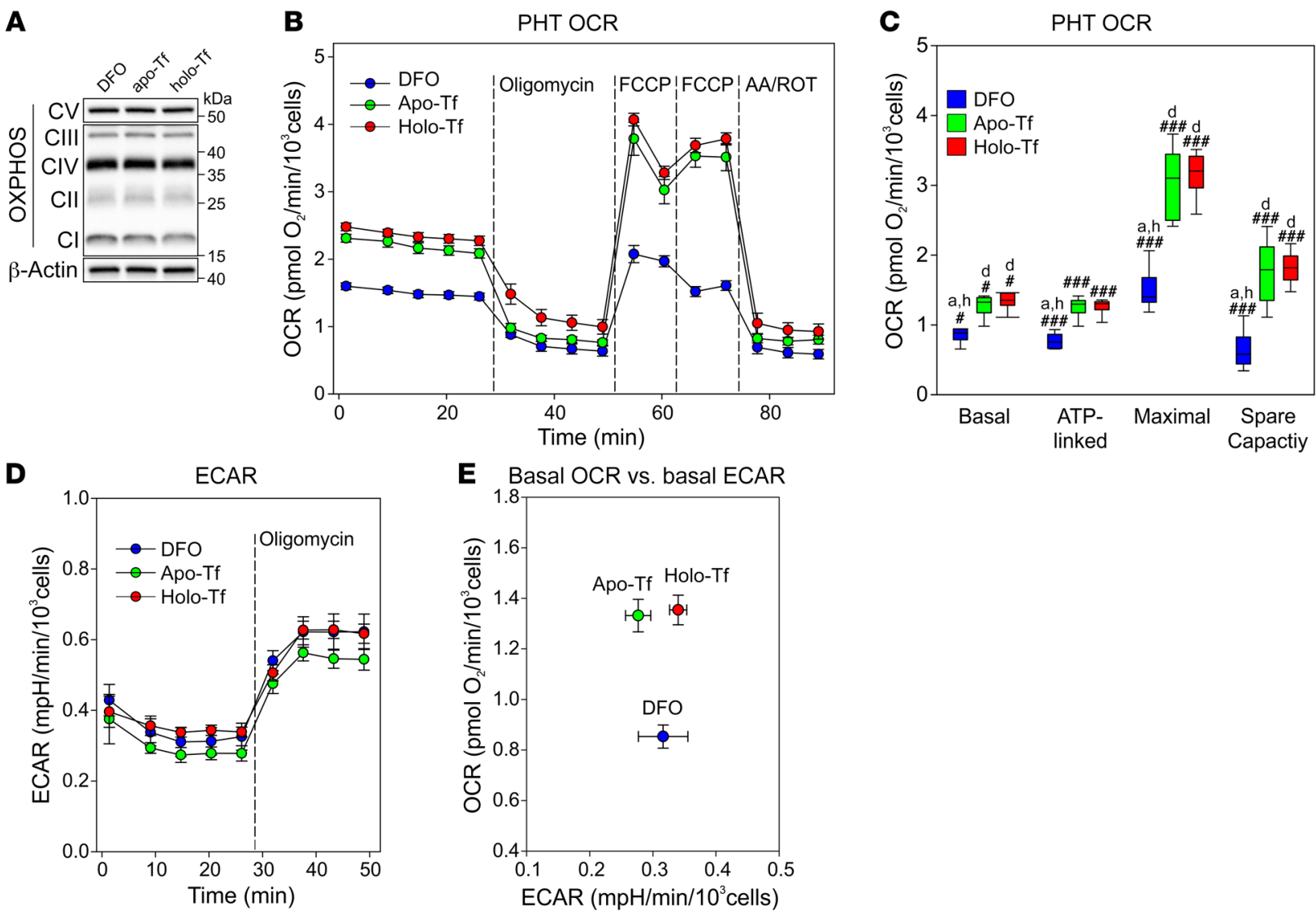

Figure 7. Iron deficiency impairs oxidative phosphorylation in PHTs. PHTs were treated for 24 hours with $100 \mu \mathrm{M}$ DFO, apo-Tf, or holo-Tf. (A) Western blotting for OXPHOS complexes CI-CV. $\beta$-Actin was used as a loading control. (B) Mitochondrial respiration under basal conditions following injection of oligomycin, the uncoupler FCCP, or the electron transport inhibitors antimycin A and rotenone (AA/ROT). (C) Quantitation of basal respiration, ATP-linked respiration, maximal respiratory capacity, and spare respiratory capacity normalized to total cells per well. Statistical differences between groups were determined by 1-way ANOVA for normally distributed values followed by an all-pairwise multiple comparison (Holm-Sidak method) $(\# \# \#<<0.001)$ or 1 -way ANOVA on ranks for non-normally distributed values followed by an all-pairwise multiple comparison (Tukey's test) ( $P<0.05)$. Lowercase letters indicate a statistical difference compared with DFO ("d"), Apo-Tf ("a"), or Holo-Tf ("h") group. $n=6$ technical replicates. (D) ECAR. (E) Basal OCR versus basal ECAR.

with a $70 \%$ average reduction in cellular ferritin levels (Supplemental Figure 9, B and C). Additionally, DFO treatment upregulated TFR1 (Supplemental Figure 9, B and D), whereas FPN was undetectable in all groups. Importantly, expression of all ETC complexes decreased in the iron-depleted cells compared with apo-Tf- or holo-Tf-treated cells (Supplemental Figure 9, E and F), suggesting that severe placental iron deficiency could impair the machinery of cellular oxidative phosphorylation.

Taken together, our data support the hypothesis that severe placental iron deficiency could compromise critical determinants of placental function, providing an evolutionary rationale for the prioritization of placental iron retention in iron-deficient pregnancy.

\section{Discussion}

Although the importance of iron for a healthy pregnancy is well recognized, how iron transfer from the mother to the fetus is regulated during pregnancy is not well understood. To determine how the maternal-placental-fetal unit responds to changes in maternal iron status, we performed a comprehensive evaluation of mechanisms in mouse models, human pregnancies, and isolated trophoblast. In mouse models, we confirmed that the maternal iron regulatory hormone hepcidin is suppressed during pregnancy, consistent with previous reports in humans (38). However, the mechanism of suppression is not yet known. We show that maternal hepcidin is regulated by maternal iron status during pregnancy, with iron-loaded mothers having higher hepcidin levels than iron-replete or irondeficient mothers. However, within each group, hepcidin was still suppressed compared with nonpregnant females, revealing a strong effect by a pregnancy-associated factor. Furthermore, reductions of both hepcidin protein and mRNA levels confirmed that maternal hepcidin suppression is not a consequence of hemodilution. Additional studies are required to determine the exact mechanism(s) of maternal hepcidin suppression during pregnancy.

Embryonic hepcidin overexpression in mice, as in transgenic hepcidin embryos or Tmprss6-deficient embryos, was reported to regulate placental FPN expression. However, in normal murine pregnancies, embryonic hepcidin expression is very low $(32,33)$. In our studies, iron endowment of the fetus was unaffected by genetic ablation of fetal and placental hepcidin in iron-replete or iron-deficient pregnancies, indicating that embryonic or placental hepcidin was too low to regulate iron transfer across the placenta under these conditions. 
Importantly, our animal models showed that during ironreplete pregnancy, homeostatic maternal adaptations - hepcidin suppression and iron mobilization from stores - maintained constant maternal serum iron levels throughout the pregnancy despite increased iron utilization in advanced pregnancy. In iron-loaded mothers, maternal hepcidin was relatively increased, preventing the release of excess iron into the maternal circulation and protecting embryos from iron overload, with only a small increase in placental iron.

In contrast to embryonic protection from maternal iron overload, maternal adaptations to iron deficiency, including the profound suppression of hepcidin, were not sufficient to protect the embryos from iron deficiency anemia. We were surprised to uncover a placental response that prioritized placental iron retention despite fetal iron deficiency. In the face of maternal iron deficiency, the murine placenta strongly decreased levels of the iron exporter FPN, resulting in decreased iron transport to the fetus. Although this ensured that the placenta developed only relatively mild iron deficiency, it was to the severe detriment of fetal iron content, with fetuses developing profound iron deficiency anemia as reflected by their low $\mathrm{Hb}$ and liver stores. In contrast to our mouse models that were stressed with severe iron deficiency, in human pregnancies that were only modestly iron deficient, expression of TFR1 protein increased but that of FPN did not change. Because human pregnancies with severe maternal iron deficiency are rare in settings in which fresh placentas can be obtained, we simulated the condition by exposing human trophoblast to a more severe iron deficiency in vitro. In agreement with the mouse model, we observed a decrease in FPN and ferritin.

We propose the PIDI, a ratio of placental iron exporter to importer (FPN/TFR1), as an indicator of fetal exposure to iron deficiency in utero. Using the ratio would minimize the effect of regional differences in human sample collection. TFR1 and FPN expression is restricted to the syncytiotrophoblast; if placental tissue collection includes an area with low or no expression of transporters, this could be erroneously interpreted as a "regulated" low expression of an individual transporter, whereas a ratio of the 2 transporters would account for the sampling variability.

Using mouse models, we also determined the mechanism underlying the altered expression of placental iron transporters following maternal iron deficiency. We demonstrated that placental IRP1 plays a critical role in sensing placental iron levels and consequent modulation of iron transporters and that $\operatorname{Irp1^{-1-}}$ placentas failed to decrease FPN protein levels in the face of maternal iron deficiency.

The ability of the placenta to retain iron for its needs prior to transport to the fetus is consistent with previous observations that when oxygen supply to the uterus is limited, the majority of the oxygen is consumed by the placenta, and oxygen transfer to the fetus is adversely affected (39). Placental retention of iron during severe iron deficiency would preserve oxidative phosphorylation to supply necessary ATP for placental protein synthesis and transport functions (36). Indeed, we show that severe iron deficiency in primary human placental trophoblast cells impaired mitochondrial respiration, whereas in the BeWo cell line, it even decreased the levels of all 5 ETC complexes. Unlike in BeWo cells, we did not observe an ETC complex decrease in primary trophoblast cells at the 24-hour time point and spec- ulate that the differences in the sensitivity of these 2 cell types to iron deficiency reflect the variability of protective cellular mechanisms against this stress. We argue that prioritization of placental iron retention in response to iron deficiency may have an evolutionary benefit: it would preserve placental iron levels even during iron deficiency so that all iron-dependent placental functions are protected, thus indirectly benefiting the fetus overall despite diminishing fetal iron availability.

Prioritization of placenta iron acquisition over iron transport to the fetus suggests that fetuses are unable to compensate for maternal iron deficiency by increasing placental iron transfer. A common misconception is that the fetus is a "perfect parasite," able to acquire adequate iron irrespective of the mother's iron status (40). However, consistent with our data, several human and macaque studies confirmed that neonatal iron stores are compromised when the mother is iron deficient or anemic (4147). Furthermore, in agreement with our model, maternal iron deficiency anemia resulted in a higher placenta/newborn weight ratio $(48,49)$. Our observation underscores the importance of detecting and treating iron-deficient pregnancies.

One of the current challenges recognized by the US Preventive Services Task Force (50) is the ability to accurately identify iron deficiency in pregnant women to determine whether iron supplementation is necessary. Currently, the most common laboratory measure is maternal $\mathrm{Hb}$, in which low $\mathrm{Hb}$ is presumed to be a result of iron deficiency; however, distinguishing actual iron deficiency anemia from $\mathrm{Hb}$ changes related to hemodilution remains a challenge (50). The lack of a correlation between maternal $\mathrm{Hb}$ and the PIDI in our human study suggests that $\mathrm{Hb}$ is not an adequate indicator of maternal iron deficiency or of the risk of fetal iron deficiency. Serum ferritin is another measure used to assess maternal iron status; although often a good indicator, this value can be confounded by infection or inflammation. In our human pregnancies, which did not have inflammation, a maternal serum ferritin level below $10 \mathrm{ng} / \mathrm{mL}$ was an accurate indicator of the risk of fetal iron deficiency as determined by the PIDI. Although hepcidin and ferritin are both acutephase reactants that respond to infection and inflammation, some studies have suggested that hepcidin is a better indicator of iron status in pregnant women than ferritin $(51,52)$. However, we found no correlation between the PIDI and maternal hepcidin levels.

Neonatal iron deficiency has been linked to numerous neurobehavioral effects (4). Most recently, an association was reported between maternal anemia diagnosed earlier in pregnancy ( $\leq 30$ weeks) and increased offspring risk of autism spectrum disorder, attention-deficit/hyperactivity disorder, and intellectual disability (6). Therefore, it is important that neonatal iron deficiency be accurately quantitated. A commonly used indicator of neonatal iron deficiency is cord blood $\mathrm{Hb}$ or cord blood ferritin. However, previous studies indicated that during fetal iron deficiency, iron is preferentially used for Hb synthesis, sacrificing neonatal brain iron endowment (53). Thus, low $\mathrm{Hb}$ may only manifest in the most severe forms of iron deficiency. Additionally, in our human study, the lack of a correlation between cord blood $\mathrm{Hb}$ or ferritin and the PIDI indicates that cord blood $\mathrm{Hb}$ and ferritin may not be sensitive indicators of neonatal iron deficiency. Therefore, we propose that the PIDI may be a superior indicator of iron deficiency in utero. As it can 
only be measured after birth, the PIDI cannot be used to guide iron supplementation during human pregnancy but may be used for future research studies to identify better markers of neonatal iron deficiency. Our study also shows that in the absence of infection or inflammation, maternal serum ferritin levels below $10 \mathrm{ng} / \mathrm{mL}$ may also signal the risk of fetal iron deficiency.

Our study demonstrates how the healthy maternal-placentalfetal unit handles iron. Maternal hepcidin is suppressed, allowing for increased absorption from the diet and release of iron from stores to maintain serum iron concentrations. This allows for optimal transfer of iron from the maternal circulation through the placenta, via TFR1 and FPN, to the fetus, where iron is then used for erythropoiesis, and any excess is stored in the fetal liver. In states of iron overload, maternal hepcidin is elevated and prevents overloading of the embryo. During iron deficiency, however, the placental homeostatic response retains iron within the placenta and protects the metabolically active placenta from severe iron deficiency and consequently decreased oxidative phosphorylation. Although this occurs at the cost of fetal iron deficiency, it may ultimately protect the fetus from more severe adverse effects of broader placental dysfunction

\section{Methods}

Mice. C57BL/6J mice were obtained from The Jackson Laboratory or bred in-house. Irp1-/ mice on a C57BL/6 background were provided by Tracey Rouault (54). Hepcidin-KO (Hamp $\left.p^{--}\right)$mice were originally provided to our laboratory by Sophie Vaulont (55) and backcrossed by us with mice on a C57BL/6 background. Unless otherwise specified, mice received a standard diet (PicoLab Rodent Diet 20, 5053 Irradiated, $185 \mathrm{ppm}$ iron) and were fed ad libitum. The iron-deficient diet (4 ppm) (TD.80396) and the iron-loaded diet (10,000 ppm, TD.08043) were purchased from Envigo Teklad Diets.

To test the effects of maternal iron status, C57BL/6J females were maintained on a standard (iron-replete) diet and then placed on an iron-deficient diet 2 weeks prior to mating and throughout gestation (iron-deficient), or received a single i.p. injection of $20 \mathrm{mg}$ iron dextran (iron loading) (MilliporeSigma, D8517) at mating. For alternative iron deficiency and loading strategies, C57BL/6J females were placed on an iron-deficient diet at mating and throughout gestation (short-term iron-deficient) or on a 10,000-ppm diet 2 weeks prior to mating and throughout gestation. Pregnant females were euthanized at the indicated gestational age, and nonpregnant female controls were euthanized on the same day.

To test the contribution of IRP1 to the maintenance of placental iron homeostasis, $\mathrm{Irp1}^{+/-}$female mice were mated with $\mathrm{Irp1}^{+ \text {-> }}$ male mice. The pregnant females were placed on an iron-deficient diet at E7.5 until E18.5, at which point they were euthanized and tissues collected for analysis.

To determine the contribution of local placental and embryonic hepcidin, $\mathrm{Hamp}^{+/}$females were maintained on an iron-replete diet or placed on an iron-deficient diet 1 week prior to mating and throughout gestation. Hamp ${ }^{+/-}$females were mated with $\mathrm{Hamp}^{+/}$- males, and pregnant females were euthanized when the embryos reached E18.5.

Human subjects. Forty-three pregnant women we recruited in their late second or early third trimester between August 2015 and October 2017 at the UIC Center for Women's Health (UI Health). Women were eligible if the following criteria were met: they had a prepregnancy BMI above $18.5 \mathrm{~kg} / \mathrm{m}^{2}$; they had naturally conceived, had a singleton pregnancy, were at 26-33 gestational weeks, and were obtaining prenatal care and planning to deliver at UI Health; were 17-45 years of age; had a parity of 0-3; were able to read and write English; were gaining the minimum amount of weight compared with their prepregnancy BMI from 23 to 24 gestational weeks onward, based on the recommendations of the Institute of Medicine; and had access to a phone to alert research staff when they were admitted to UI Health for labor and delivery. The following criteria were used to exclude women from this study: live birth or other pregnancy (including ectopic and molar pregnancies) in the previous 12 months; autoimmune disorder; gestational diabetes mellitus or previously diagnosed diabetes; current or previous premature rupture of membranes or chorioamnionitis; previous spontaneous premature birth; current bacterial or viral infection; current steroid or antiinflammatory treatment; previous bariatric surgery; malabsorptive disease; current hyperemesis; hematologic disorder (i.e., sickle cell disease, sickle cell trait, or hemochromatosis); tobacco use in the past 3 months; current alcohol consumption; or current illicit drug use.

One blood sample was collected at 32-34 gestational weeks. Women were asked to fast for 1.5 hours and refrain from all vitamin and mineral supplements for 48 hours prior to the visit. Upon admission to the UI Health Labor and Delivery facility (at $38.9 \pm 1.4$ weeks), an additional maternal blood sample was obtained. Placentas were processed within 30 minutes of delivery. The placenta was visually divided into quadrants, a was core sampled from each quadrant and sectioned into smaller pieces, and pieces from all 4 cores were combined to provide a representative sample of the tissue. Aliquots were either snap-frozen, or RNAlater (Ambion) was added.

Cell culture. PHTs were prepared from normal-term placentas using a modified trypsin-deoxyribonuclease-dispase/Percoll method $(56,57)$. PHTs were cultured in DMEM plus 10\% FBS. BeWo cells obtained from the American Type Culture Collection (ATCC) (CCL98) were cultured in Ham's F-12K Nutrient Mixture plus 10\% FBS. For in vitro iron depletion and iron-loading experiments, PHTs were seeded at $1 \times 10^{6} /$ well and BeWo cells at $1 \times 10^{5} /$ well in a 6 -well, collagen-coated plate (Corning BioCoat), allowed to attach overnight, and treated with $100 \mu \mathrm{M}$ DFO (MilliporeSigma, D9533), $100 \mu \mathrm{M}$ apo-Tr (Celliance, 4452-01), or $100 \mu \mathrm{M}$ holo-Tf (MilliporeSigma, 4455-01) for 24 hours.

Complete blood counts. Mouse complete blood counts (CBCs) were performed on a Hemavet 950FS hematology system (Drew Scientific). Human $\mathrm{Hb}$ was determined using an Abbott HemoCue point-of-care monitor.

Iron measurements. Serum, hepatic, and placental nonheme iron concentrations were measured using a previously described method (58) (Sekisui Diagnostics, 157-30). Human maternal and neonatal cord serum iron and serum ferritin were measured at Quest Diagnostics. The unsaturated iron-binding capacity (UIBC) was measured using a Pointe Scientific assay (I7504). The transferrin saturation percentage $=($ serum iron $/$ total iron-binding capacity $[\mathrm{TIBC}]) \times 100$.

${ }^{58} \mathrm{Fe}$ isotope preparation and sample analysis. ${ }^{58} \mathrm{Fe}$ (93\% enrichment) was purchased from Trace Sciences International, dissolved in $12 \mathrm{~N} \mathrm{HCl}$ to form $\mathrm{H}_{2}$ plus ${ }^{58} \mathrm{FeCl}_{2}$, and $\mathrm{H}_{2} \mathrm{O}_{2}$ was added to oxidize the $\mathrm{H}_{2}$ plus ${ }^{58} \mathrm{FeCl}_{2}$ to $\mathrm{H}_{2} \mathrm{O}$ plus ${ }^{58} \mathrm{FeCl}_{3}{ }^{58}{ }^{5 e C l}{ }_{3}$ was incubated with nitrilotriacetate (NTA) at a molar ratio of 1:5 in $20 \mathrm{mM} \mathrm{NaHCO}_{3}$ for 5 minutes at room temperature to form ${ }^{58} \mathrm{Fe}-\mathrm{NTA}$. Human apo-Tf (Cel- 
liance, 4452-01) was dissolved in Tf-loading buffer (0.1 M HEPES, $\mathrm{pH} 7.5,0.15 \mathrm{M} \mathrm{NaCl}$ ) and incubated with ${ }^{58} \mathrm{Fe}-\mathrm{NTA}$ at a molar ratio of 1:2 for 2.5 hours at room temperature to form ${ }^{58} \mathrm{Fe}$-Tf. To remove unbound ${ }^{58} \mathrm{FeCl}_{3}$, the ${ }^{58} \mathrm{Fe}$-Tf mixture was centrifuged in an Amicon Ultra Filter $30 \mathrm{~K}$ at $2500 \mathrm{~g}$ for 15 minutes and washed with Tf-loading buffer and saline. Each pregnant mouse received a retro-orbital injection of $3.5 \mathrm{mg}$ human ${ }^{58} \mathrm{Fe}$-Tf (equivalent to $5 \mu \mathrm{g}{ }^{58} \mathrm{Fe}$ ) at E17.5. Tissues were collected 6 hours after injection. Placentas and fetal livers were processed for nonheme iron as previously described (58), and supernatant was submitted for ICP-MS analysis of ${ }^{56} \mathrm{Fe}$ and ${ }^{58} \mathrm{Fe}$. The remaining iron (mostly heme) was extracted from tissue pellets by digestion in $\mathrm{HNO}_{3}$ supplemented with $\mathrm{H}_{2} \mathrm{O}_{2}$, the samples were heated up to $200^{\circ} \mathrm{C}$ for 15 minutes followed by dilution of samples with $2 \% \mathrm{HNO}_{3}$, and the samples were analyzed by ICP-MS. The sum of nonheme and heme iron was presented as the total iron content. For ${ }^{58} \mathrm{Fe}$ measurements, the natural abundance $(0.28 \%$ of total $\mathrm{Fe})$ was subtracted from the measured ${ }^{58} \mathrm{Fe}$ values.

Serum hepcidin. Mouse serum hepcidin levels were measured using a sandwich ELISA by Amgen (59).

Quantitative PCR. RNA was prepared using TRIzol (Invitrogen, Thermo Fisher Scientific), and cDNA was synthesized using an iScript cDNA Synthesis Kit (Bio-Rad). Quantitative PCR (qPCR) was performed with SsoAdvanced SYBR Green Supermix (Bio-Rad) (primers are listed in Supplemental Table 5). The samples were run in duplicate on a CFXconnect qPCR instrument (Bio-Rad). miR-485-3p was measured using a QIAGEN miScript Primer Assay (MSO0006335).

Immunofluorescence. Formalin-fixed, paraffin-embedded sections $(5-\mu \mathrm{m})$ of mouse and human placentas were used. Tris-EDTA buffer (10 mM Tris, $1 \mathrm{mM}$ EDTA, $\mathrm{pH}$ 9.0) was used for antigen retrieval, and sections were heated to $96^{\circ} \mathrm{C}$ for 10 minutes. Primary antibodies (Supplemental Table 6) were incubated overnight at room temperature in a humidified chamber and secondary antibodies for 1 hour at room temperature. Images were captured using a Zeiss LSM 700 confocal microscope.

Western blot analysis. Tissues were lysed by homogenization in RIPA buffer and cells by addition of RIPA (Santa Cruz Biotechnology, sc-24948), and lysates were cleared by centrifugation at $17,000 \mathrm{~g}$ for 15 minutes at $4^{\circ} \mathrm{C}$. Protein was quantified using a Pierce BCA Assay (Thermo Fisher Scientific, 23225). Samples for FPN and OXPHOS were prepared in Laemmli sample buffer without a reducing agent, and samples were not boiled. For all other proteins, samples were prepared in Laemmli buffer with DTT and incubated at $100^{\circ} \mathrm{C}$ for 5 minutes. The samples were resolved on Bio-Rad 4\%-20\% TGX gels or Any kD TGX gels for OXPHOS, electroblotted onto nitrocellulose (Trans-Blot Turbo System, Bio-Rad), and imaged with a ChemiDoc XRS+ system (Bio-Rad). The primary and secondary antibodies used are listed in Supplemental Table 6. Membranes were stripped using $0.2 \mathrm{~N} \mathrm{NaOH}$ for 10 minutes at room temperature and reprobed for the loading controls. Quantitation was performed using Image Lab Software, version 5.2.1 (Bio-Rad).

PIDI calculation. Since mouse FPN and TFR1 expression was analyzed on separate Western blots, given the need for different handling of the samples, we first normalized each of the proteins to their $\beta$-actin loading control and then generated the PIDI as the ratio of normalized FPN to normalized TFR1. In human placenta, because several electrophoresis gels had to be used to analyze the large number of samples, TFR1, FPN, and $\beta$-actin values were normalized to the median for each membrane to account for intermembrane variability, and then the values were divided by the normalized $\beta$-actin values to account for any loading variation. The normalized FPN values were then divided by the normalized TFR1 values to generate the PIDI.

$E M S A$. The direct interaction between placental IRP1 and IRP2 and IREs was determined using a LightShift Chemiluminescent RNA EMSA Kit (Thermo Fisher Scientific, 20158). Whole placenta tissue lysate $(4 \mu \mathrm{g})$ was used for each reaction, and samples were then resolved on a pre-cast 6\% DNA retardation gel (Thermo Fisher Scientific, EC6365BOX) and transferred onto a Biodyne B nylon membrane (Thermo Fisher Scientific, 77016) using a Trans-Blot Turbo Transfer System (Bio-Rad) at $200 \mathrm{~mA}$ for 30 minutes. Transferred RNA was crosslinked onto the membrane using the "autocrosslink" function on the Stratagene Stratalinker UV Crosslinker 2400. RNA detection was performed using a Chemiluminescent Nucleic Acid Detection Module (Thermo Fisher Scientific, 89880), and membranes were imaged on a ChemiDoc XRS+.

Respirometry assays. The OCR and ECAR were measured using the Seahorse XFe96 flux analyzer (Agilent Technologies). PHTs (40,000/ well or 60,000/well) were plated onto a Seahorse XF96 cell culture plate (Agilent Technologies, 101085-004) coated with collagen (MilliporeSigma, 125-50) and were then cultured for 3 hours to allow attachment, washed 3 times with PBS, and cultured in DMEM plus 1\% FBS supplemented with $100 \mu \mathrm{M}$ DFO, $100 \mu \mathrm{M}$ apo-Tf, or $100 \mu \mathrm{M}$ holoTf for 24 hours. At the time of the assay, the treatment medium was removed and replaced with the appropriate prewarmed Seahorse XF Assay Medium (Agilent Technologies) consisting of DMEM containing $10 \mathrm{mM}$ glucose and $4 \mathrm{mM}$ glutamine. Cells were then subjected to a Mitochondria stress test (Agilent Technologies). Oxygen consumption measurements were made approximately every 5 minutes under basal conditions, after the addition of $2.5 \mu \mathrm{M}$ oligomycin, $1 \mu \mathrm{M}$ carbonyl cyanide-4-(trifluoromethoxy) phenylhydrazone (FCCP), and 2 $\mu \mathrm{M}$ antimycin A and rotenone. OCR and ECAR measurements were normalized to the number of cells per well following the assay. The contribution from nonmitochondrial respiration as measured following antimycin A and rotenone injection was subtracted from all raw OCR values. Basal OCRs were determined at the last measurement prior to the injection of oligomycin. ATP-linked respiration was determined as the difference between the oligomycin-resistant OCR and basal respiration. Maximal respiration was determined as the highest value after the addition of FCCP. Spare capacity was determined by the difference between maximal FCCP and the basal OCR. ECAR measurements were performed in parallel. Basal ECARs were determined at the last measurement prior to the injection of oligomycin. The post-oligomycin maximum ECAR was the calculated average of 4 measurements taken after oligomycin injection.

Statistics. For box plots, the upper portion of the box plot indicates the $75^{\text {th }}$ percentile and the bottom indicates the $25^{\text {th }}$ percentile, whiskers above the box indicate the $90^{\text {th }}$ percentile, those below the box indicate the tenth percentile, and individual points represent outliers. The solid line within the box indicates the median and the dashed line the mean. Statistical analysis was performed using SigmaPlot scientific graphing and data analysis software (Systat Software). A P value of less than 0.05 was considered statistically significant. Statistical differences between groups were determined using a 1-way ANOVA for normally distributed values followed by the Holm-Sidak method for multiple comparisons; 
a 1-way ANOVA on ranks followed by Dunn's method for multiple comparisons for non-normally distributed values; a 2-tailed, unpaired Student's $t$ test for normally distributed values; a Mann-Whitney $U$ for non-normally distributed values; or a 2-way ANOVA.

Study approval. All study procedures were approved by the UIC Institutional Review Board (approval no. 2015-0353), and all participants provided written informed consent.

\section{Author contributions}

VS designed and performed experiments, analyzed data, and wrote the manuscript. ALF performed experiments and assisted with data interpretation. SW assisted with experiments. MDK and LTH enrolled the study participants and collected human data and samples and edited the manuscript. AC participated in studies using PHTs and edited the manuscript. ML assisted with human placenta studies and method development and edited the manuscript. TG and EN conceived the project, analyzed data, and wrote the manuscript.

\section{Acknowledgments}

The authors thank Deliang Zhang and Tracey Rouault for providing Irp1-KO mice; the UCLA Translational Pathology Core laboratory for histology processing; the UCLA Broad Stem Cell Center
Microscopy Core for providing access to and training on confocal microscopes; Linsey Stiles and Brandon Desousa at the UCLA Mitochondria and Metabolism Core for running the respirometry assays; the UCLA ICP-MS core facility within the UC Center for Environmental Implications of Nanotechnology; Tiffany Coon and Yoel Sadovsky at the Magee-Womens Research Institute for their assistance with primary trophoblast isolation; and Victoria Gabayan and Erika Valore for their assistance with methods development. The study was supported by the Executive Advisory Board of the Iris Cantor-UCLA Women's Health Center and National Center for Advancing Translational Science (NCATS) pilot grants (UCLA CTSI grant UL1TR000124, to EN and VS); an NIH Ruth L. Kirschstein National Research Service Award (T325T32HL072752-13, to VS); a Robert Wood Johnson Foundation, Nurse Faculty Scholars Award (72117, to MDK); a UIC College of Nursing Dean's Fund grant (to MDK and LTH); and a National Institute of Child Health and Human Development (NICHD) NIH grant (R01HD096863, to EN).

Address correspondence to: Elizabeta Nemeth, UCLA David Geffen School of Medicine, 10833 LeConte Ave, CHS 43-229, Los Angeles, California 90095, USA. Phone: 310.825.2841; Email: ENemeth@mednet.ucla.edu.
1. Fisher AL, Nemeth E. Iron homeostasis during pregnancy. Am J Clin Nutr. 2017;106(Suppl 6):1567S-1574S

2. Ezzati M, Lopez AD, Rodgers A, Vander Hoorn S, Murray CJ, Comparative Risk Assessment Collaborating Group. Selected major risk factors and global and regional burden of disease. Lancet. 2002;360(9343):1347-1360.

3. Daru J, et al. Risk of maternal mortality in women with severe anaemia during pregnancy and post partum: a multilevel analysis. Lancet Glob Health. 2018;6(5):e548-e554.

4. Georgieff MK. Long-term brain and behavioral consequences of early iron deficiency. Nutr Rev. 2011;69(Suppl 1):S43-S48.

5. Scholl TO, Reilly T. Anemia, iron and pregnancy outcome. J Nutr. 2000;130(2S Suppl):443S-447S.

6. Wiegersma AM, Dalman C, Lee BK, Karlsson H, Gardner RM. Association of prenatal maternal anemia with neurodevelopmental disorders. JAMA Psychiatry. 2019;76(12):1-12.

7. World Health Organization. Guideline: Daily Iron and Folic Acid Supplementation in Pregnant Women. Geneva, Switzerland: World Health Organization; 2012.

8. Taylor CL, Brannon PM. Introduction to workshop on iron screening and supplementation in iron-replete pregnant women and young children. Am J Clin Nutr. 2017;106(Suppl 6):1547S-1554S

9. Wardman P, Candeias LP. Fenton chemistry: an introduction. Radiat Res. 1996;145(5):523-531.

10. Ganz T. Iron and infection. Int J Hematol. 2018;107(1):7-15.

11. Lao TT, Tam KF, Chan LY. Third trimester iron status and pregnancy outcome in non-anaemic women; pregnancy unfavourably affected by maternal iron excess. Hum Reprod.
2000;15(8):1843-1848.

12. Dewey KG, Oaks BM. U-shaped curve for risk associated with maternal hemoglobin, iron status, or iron supplementation. Am J Clin Nutr. 2017;106(Suppl 6):1694S-1702S.

13. Ganz T. Systemic iron homeostasis. Physiol Rev. 2013;93(4):1721-1741.

14. Bastin J, Drakesmith H, Rees M, Sargent I, Townsend A. Localisation of proteins of iron metabolism in the human placenta and liver. $\mathrm{Br}$ Haematol. 2006;134(5):532-543.

15. Donovan A, et al. Positional cloning of zebrafish ferroportin1 identifies a conserved vertebrate iron exporter. Nature. 2000;403(6771):776-781.

16. Levy JE, Jin O, Fujiwara Y, Kuo F, Andrews NC. Transferrin receptor is necessary for development of erythrocytes and the nervous system. Nat Genet. 1999;21(4):396-399.

17. Donovan A, et al. The iron exporter ferroportin/ Slc40a1 is essential for iron homeostasis. Cell Metab. 2005;1(3):191-200.

18. Gambling L, et al. Effect of iron deficiency on placental transfer of iron and expression of iron transport proteins in vivo and in vitro. Biochem $\mathrm{J}$. 2001;356(Pt 3):883-889.

19. Li YQ, Yan H, Bai B. Change in iron transporter expression in human term placenta with different maternal iron status. Eur JObstet Gynecol Reprod Biol. 2008;140(1):48-54.

20. Muckenthaler MU, Galy B, Hentze MW. Systemic iron homeostasis and the iron-responsive element/iron-regulatory protein (IRE/IRP) regulatory network. Annu Rev Nutr. 2008;28:197-213.

21. Ghosh MC, Zhang DL, Rouault TA. Iron misregulation and neurodegenerative disease in mouse models that lack iron regulatory proteins. Neurobiol Dis. 2015;81:66-75.

22. van Santen S, Kroot JJ, Zijderveld G, Wiegerinck
ET, Spaanderman ME, Swinkels DW. The iron regulatory hormone hepcidin is decreased in pregnancy: a prospective longitudinal study. Clin Chem Lab Med. 2013;51(7):1395-1401.

23. van Santen S, Kroot JJ, Zijderveld G, Wiegerinck ET, Spaanderman ME, Swinkels DW. The iron regulatory hormone hepcidin is decreased in pregnancy: a prospective longitudinal study. Clin Chem Lab Med. 2013;51(7):1395-1401.

24. Cao C, Fleming MD. The placenta: the forgotten essential organ of iron transport. Nutr Rev. 2016;74(7):421-431.

25. Yoshinaga M, et al. Regnase-1 maintains iron homeostasis via the degradation of transferrin receptor 1 and prolyl-hydroxylasedomain-containing protein 3 mRNAs. Cell Rep 2017;19(8):1614-1630.

26. Cianetti L, et al. Expression of alternative transcripts of ferroportin-1 during human erythroid differentiation. Haematologica. 2005;90(12):1595-1606.

27. Zhang DL, Hughes RM, Ollivierre-Wilson $\mathrm{H}$, Ghosh MC, Rouault TA. A ferroportin transcript that lacks an iron-responsive element enables duodenal and erythroid precursor cells to evade translational repression. Cell Metab. 2009;9(5):461-473.

28. Georgieff MK, Berry SA, Wobken JD, Leibold EA. Increased placental iron regulatory protein-1 expression in diabetic pregnancies complicated by fetal iron deficiency. Placenta. 1999;20 (1):87-93.

29. Bradley J, et al. Influence of gestational age and fetal iron status on IRP activity and iron transporter protein expression in third-trimester human placenta. Am J Physiol Regul Integr Comp Physiol. 2004;287(4):R894-R901.

30. Smith SR, Ghosh MC, Ollivierre-Wilson H, Hang Tong W, Rouault TA. Complete loss of iron 
regulatory proteins 1 and 2 prevents viability of murine zygotes beyond the blastocyst stage of embryonic development. Blood Cells Mol Dis. 2006;36(2):283-287.

31. Lakhal-Littleton S, et al. An essential cell-autonomous role for hepcidin in cardiac iron homeostasis. Elife. 2016;5:e19804.

32. Nicolas G, et al. Severe iron deficiency anemia in transgenic mice expressing liver hepcidin. Proc Natl Acad Sci USA. 2002;99(7):4596-4601.

33. Willemetz A, et al. Matriptase- 2 is essential for hepcidin repression during fetal life and postnatal development in mice to maintain iron homeostasis. Blood. 2014;124(3):441-444.

34. Sangokoya C, Doss JF, Chi JT. Iron-responsive miR-485-3p regulates cellular iron homeostasis by targeting ferroportin. PLoS Genet. 2013;9(4):e1003408.

35. Bonds DR, Crosby LO, Cheek TG, Hägerdal M, Gutsche BB, Gabbe SG. Estimation of human fetal-placental unit metabolic rate by application of the Bohr principle. J Dev Physiol. 1986;8(1):49-54.

36. Rolfe DF, Brown GC. Cellular energy utilization and molecular origin of standard metabolic rate in mammals. Physiol Rev. 1997;77(3):731-758.

37. Heaton SJ, et al. The use of BeWo cells as an in vitro model for placental iron transport. Am J Physiol, Cell Physiol. 2008;295(5):C1445-C1453.

38. Koenig MD, Tussing-Humphreys L, Day J, Cadwell B, Nemeth E. Hepcidin and iron homeostasis during pregnancy. Nutrients. 2014;6(8):3062-3083.

39. Carter AM. Placental oxygen consumption. Part I: in vivo studies--a review. Placenta. 2000;21(Suppl A):S31-S37.

40. Allen LH. Anemia and iron deficiency: effects on pregnancy outcome. Am JClin Nutr. 2000; 71(5 Suppl):1280S-1284S.

41. Radlowski EC, Johnson RW. Perinatal iron defi- ciency and neurocognitive development. Front Hum Neurosci. 2013;7:585.

42. Scholl TO. Maternal iron status: relation to fetal growth, length of gestation, and iron endowment of the neonate. Nutr Rev. 2011;69(Suppl 1):S23-S29.

43. Jaime-Perez JC, Herrera-Garza JL, GomezAlmaguer D. Sub-optimal fetal iron acquisition under a maternal environment. Arch Med Res. 2005;36(5):598-602.

44. Lubach GR, Coe CL. Preconception maternal iron status is a risk factor for iron deficiency in infant rhesus monkeys (Macaca mulatta). J Nutr. 2006;136(9):2345-2349.

45. Colomer J, et al. Anaemia during pregnancy as a risk factor for infant iron deficiency: report from the Valencia Infant Anaemia Cohort (VIAC) study. Paediatr Perinat Epidemiol. 1990;4(2):196-204.

46. Kilbride J, Baker TG, Parapia LA, Khoury SA, Shuqaidef SW, Jerwood D. Anaemia during pregnancy as a risk factor for iron-deficiency anaemia in infancy: a case-control study in Jordan. Int J Epidemiol. 1999;28(3):461-468.

47. De Pee S, Bloem MW, Sari M, Kiess L, Yip R, Kosen S. The high prevalence of low hemoglobin concentration among Indonesian infants aged 3-5 months is related to maternal anemia. J Nutr. 2002;132(8):2215-2221.

48. Larsen S, Bjelland EK, Haavaldsen C, Eskild A. Placental weight in pregnancies with high or low hemoglobin concentrations. Eur JObstet Gynecol Reprod Biol. 2016;206:48-52.

49. Lao TT, Tam KF. Placental ratio and anemia in third-trimester pregnancy. J Reprod Med. 2000;45(11):923-928.

50. Cantor AG, Bougatsos C, Dana T, Blazina I, McDonagh M. Routine iron supplementation and screening for iron deficiency anemia in pregnancy: a systematic review for the U.S.
Preventive Services Task Force. Ann Intern Med. 2015;162(8):566-576.

51. Bah A, et al. Serum hepcidin concentrations decline during pregnancy and may identify iron deficiency: analysis of a longitudinal pregnancy cohort in the Gambia. J Nutr. 2017;147(6):1131-1137.

52. Schulze KJ, et al. Hepcidin and iron status among pregnant women in Bangladesh. Asia Pac JClin Nutr. 2008;17(3):451-456.

53. Lozoff B, Georgieff MK. Iron deficiency and brain development. Semin Pediatr Neurol. 2006;13(3):158-165.

54. Meyron-Holtz EG, et al. Genetic ablations of iron regulatory proteins 1 and 2 reveal why iron regulatory protein 2 dominates iron homeostasis. EMBO J. 2004;23(2):386-395.

55. Lesbordes-Brion JC, et al. Targeted disruption of the hepcidin 1 gene results in severe hemochromatosis. Blood. 2006;108(4):1402-1405.

56. Nelson DM, Johnson RD, Smith SD, Anteby EY, Sadovsky Y. Hypoxia limits differentiation and up-regulates expression and activity of prostaglandin $\mathrm{H}$ synthase 2 in cultured trophoblast from term human placenta. Am JObstet Gynecol. 1999;180(4):896-902.

57. Kliman HJ, Nestler JE, Sermasi E, Sanger JM, Strauss JF. Purification, characterization, and in vitro differentiation of cytotrophoblasts from human term placentae. Endocrinology. 1986;118(4):1567-1582.

58. Stefanova D, et al. Endogenous hepcidin and its agonist mediate resistance to selected infections by clearing non-transferrin-bound iron. Blood. 2017;130(3):245-257.

59. Kim A, et al. A mouse model of anemia of inflammation: complex pathogenesis with partial dependence on hepcidin. Blood. 2014;123(8):1129-1136. 